\title{
L'impact des prélèvements d'eau pour l'irrigation sur les régimes hydrologiques des sous-bassins du Tescou et de la Séoune (bassin Adour-Garonne, France) Water withdrawn from Tescou and Seoune rivers for the irrigation, its consequences on the hydrological regime of the sub-basins (Adour-Garonne watershed, France)
}

\author{
G. Galéa, B. Vasquez-Paulus, B. Renard et P. Breil
}

Volume 18, numéro 3, 2005

URI : https://id.erudit.org/iderudit/705560ar

DOI : https://doi.org/10.7202/705560ar

Aller au sommaire du numéro

Éditeur(s)

Université du Québec - INRS-Eau, Terre et Environnement (INRS-ETE)

ISSN

0992-7158 (imprimé)

1718-8598 (numérique)

Découvrir la revue

Citer cet article

Galéa, G., Vasquez-Paulus, B., Renard, B. \& Breil, P. (2005). L'impact des prélèvements d'eau pour l'irrigation sur les régimes hydrologiques des sous-bassins du Tescou et de la Séoune (bassin Adour-Garonne, France). Revue des sciences de l'eau / Journal of Water Science, 18(3), 273-305.

https://doi.org/10.7202/705560ar

\section{Résumé de l'article}

Les besoins en eau pour l'irrigation des cultures des sous-bassins du Tescou et de la Séoune (Bassin Adour-Garonne) se sont considérablement accrus ces trente dernières années. Cela s'est manifesté, entre autres, par la création de très nombreuses retenues collinaires. Pour le sous-bassin du Tescou, aux crue rapides et peu volumineuses, on totalise 184 retenues individuelles dont le volume théorique cumule s'élève à $4,3 \mathrm{Mm}^{3}$ et qui interceptent environ un tiers $\left(92 \mathrm{~km}^{2}\right)$ de sa superficie $\left(287 \mathrm{~km}^{2}\right)$. Pour la Séoune, la pression due à l'usage de l'eau pour l'irrigation est un peu moins forte, compte tenu de sa taille $\left(463 \mathrm{~km}^{2}\right)$ et d'une ressource relativement abondante en hautes eaux. Près de 160 retenues collinaires sont dénombrées, elle représentent un volume de stockage théorique de $6,5 \mathrm{Mm}^{3}$ pour une superficie interceptée d'environ un quart du sous-bassin $\left(122 \mathrm{~km}^{2}\right)$. Le suivi hydrométrique des sous-bassins permet de disposer pour chacun d'eux d'une chronique de débit influencée $Q(t)$ sur une période d'observation d'environ 30 ans. A partir des débits observés, notre objectif a été d'identifier et de quantifier l'impact anthropique pour trois composantes du régime hydrologique : crue, module et étiage. L'approche saisonnière des crues et modules composantes du régime hydrologique : crue, module et étiage. L'approche saisonnière des crues et modules a permis une differenciation de l'impact anthropique selon les saisons "hiver" (mois 12 à 6) et "éte" (mois 7 ces sous-bassins agricoles : stockage l'hiver et irrigation à partir des retenues collinaires durant les ces sous-bassins agricoles : stockage l'hiver et irrigation à partir des retenues collinaires durant les
premiers mois d'été. Pour identifier l'impact, nous avons fait usage de tests de détection de rupture de premiers mois d'été. Pour identifier l'impact, nous avons fait usage de tests de détection de rupture de BOIS (1986) pour les modules et étiages des sous-bassins. Pour ce qui concerne les crues d'été et les modules d'été les tests montrent que les chroniques sont stationnaires sur l'ensemble de la période d'observation. d'été les tests montrent que les chroniques sont stationnaires sur l'ensemble de la période d'observation. chroniques qui vont nous permettre de définir des sous-périodes stationnaires. Ces sous-périodes son sensiblement cohérentes avec l'évolution des prélèvements théoriques cumulés de ces trente dernières années. L'analyse des ruptures de stationnarité menée sur les débits a été de même effectuée sur les pluies pour consolider nos résultats. La stationnarité des chroniques de pluie observées montre qu'il n'y a pas de composante climatique, autrement dit que la tendance détectée sur les débits est essentiellement d'origine anthropique. Il ne semble pas y avoir d'impact des éventuels pompages sur les débits d'étiage et tout particulièrement pour ce qui concerne la norme VCN30 "débit moyen minimum annuel sur 30 jours consécutifs" des deux sous-bassins. Finalement, l'usage de l'eau pour l'irrigation affecte essentiellement les crues et les modules de la saison hiver. Un essai de quantification de cette tendance a été menée à partir d'une analyse statistique asociant les observations des sous-périodes stationnaires et les débits simulés

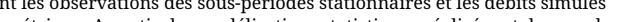
pour un memes reurbes de pression des besins theoriques de linigation, quantiles de crue et les moddles dhiver. De maniere generale, les crues de la saison hiver des deux sous-bassins sont fortement réduites en pointe et volume par l'ensemble des petites retenues. L'impact observé sur les quantiles de crue reste coherent par rapport aux volumes theoriques prélevés dernièrement référencés. Les retenues collinaires sont sans action significative sur le temps de transfert (19j environ) du sous-bassin de la Séoune dont les crues sont volumineuses. Pour le Tescou par contre, aux crues rapides et peu volumineuses, le temps de transfert augmente sensiblement de $8 \mathrm{~h}$ entre l'état de prélèvement zéro ("naturel" : 1,73j) et l'état actuel proche de $6 \mathrm{Mm}^{3}(2,07 j)$. Pour ce qui concerne les modules d'hiver des sous-bassins de la Séoune et du Tescou le coefficient d'écoulement moyen d'hive diminue respectivement de $31 \%$ en moyenne et de $42 \%$ en moyenne entre l'état "naturel" et l'état anthropisé actuel. Cette diminution des coefficients d'écoulement est sans commune mesure avec les volumes théoriques stockés à partir des retenues collinaires. Autrement dit, les volumes théoriques des retenues collinaires ne peuvent expliquer à eux seuls la forte diminution des coefficients d'écoulement observés. Cependant, la cohérence des divers contrôles effectués, tant sur les chroniques observées que sur observes. Cependant, la cohérence des divers contrôles effectues, tant sur les chroniques observées que sur objectifs de gestion immédiats ou ultérieurs de la ressource, si les conditions climatiques et d'occupation du sol n'évoluent guère.
Tous droits réservés @ C Revue des sciences de l'eau, 2005
Ce document est protégé par la loi sur le droit d'auteur. L'utilisation des services d'Érudit (y compris la reproduction) est assujettie à sa politique d'utilisation que vous pouvez consulter en ligne.

https://apropos.erudit.org/fr/usagers/politique-dutilisation/ 


\title{
L'impact des prélèvements d'eau pour l'irrigation sur les régimes hydrologiques des sous-bassins du Tescou et de la Séoune (bassin Adour-Garonne, France)
}

\author{
Water withdrawn from Tescou and Seoune rivers \\ for the irrigation, its consequences on \\ the hydrological regime of the sub-basins \\ (Adour-Garonne watershed, France)
}

G. GALEA ${ }^{1 \star}$, S. VASQUEZ-PAULUS ${ }^{2}$, B. RENARD ${ }^{1}$, P. BREIL ${ }^{1}$

Reçu le 15 novembre 2004, accepté le 27 avril 2005**.

SUMMARY

The water needs for farming irrigation in the Tescou and Séoune sub-basins (Basin Adour-Garonne) have increased considerably over the past thirty years. The needs were met, in part, by the creation of numerous reservoirs. For the Tescou catchment, with rapid but small-volume floods, we have 184 individual reservoirs with an accumulated theoretical volume of $4.3 \mathrm{Mm}^{3}$, which intercept about a third $\left(92 \mathrm{~km}^{2}\right)$ of its surface $\left(287 \mathrm{~km}^{2}\right)$. For the Séoune catchment, the need for irrigation is a little less strong, considering its size $\left(463 \mathrm{~km}^{2}\right)$ and the presence of a relatively more abundant water resource. Close to 160 reservoirs can be counted, with a theoretical storage volume of $6.5 \mathrm{Mm}^{3}$ for an intercepted surface of about a quarter of the subbasin $\left(122 \mathrm{~km}^{2}\right)$. Hydrological monitoring data are available in the form of an influenced time series $Q(t)$ with a period of observation of about 30 years for each sub-basin. From the observed discharges, our objective was to identify and to quantify anthropogenic impacts on three components of the hydrological regime: floods, annual mean discharge and low-flows. The seasonal approach of floods and annual mean discharges permitted a differentiation of the anthropogenic impact according to the "winter" (months 12 to 6) and "summer" (months 7 to 11) seasons. This approach reflects the present management of water in these agricultural sub-basins: storage in winter and irrigation during the first months of summer. To identify the impact, we used tests to detect stationnarity breaks in the time series: LANG (2004) for floods and an adaptation of BoIS (1986) for annual mean discharges and low-flows.

1. Cemagref-Lyon, Unité de Recherche Hydrologie Hydraulique, 3 bis, quai Chauveau 69336, Lyon Cedex 09, France.

2. Ingénieur Civil, Université du Chili, Faculté des Sciences Physiques et Mathématiques, Blanco Encalada 2002, Santiago, Chili.

* Correspondance: gilles.galea@cemagref.fr

* * Les commentaires seront reçus jusqu'au 30 juin 2006. 
For summer floods and summer annual mean discharges, the tests showed that the time series were stationary during the period of observation. For winter floods and winter annual mean discharges the tests showed stationnarity breaks in the time series, which allowed us to define the stationary sub-periods. These sub-periods were consistent with the evolution of the accumulated theoretical withdrawals during the last thirty years. The same analysis of stationnarity breaks made on flows was also done on rainfall to strengthen our results. The stationnarity of the rainfall observed over the time series shows that there is not a climatic component; in other words, the trends detected in the streamflow record are essentially of anthropogenic origin. For both sub-basins, the Bors statistical test did not reveal any impact of possible pumping on the low-flows, and especially for the VCN30 norm (minimum annual mean flow over a period of 30 consecutive days).

Finally, the use of water for irrigation essentially affects winter floods and annual mean discharges. A test to quantify this trend was carried out with a statistical analysis associating the observations of each stationary sub-period and the discharges simulated for a similar rainfall. From the resulting statistical models and the curves of pressure of theoretical irrigation requirements, we deduced a probable trend for the quantiles of winter floods and mean annual winter discharges. In a general way, winter floods in both sub-basins were strongly reduced in peak and volume by the set of the small reservoirs. The impact observed on the flood quantiles remains coherent in relation to the theoretical withdrawal volumes referenced above. The reservoirs are without significant action on the transfer time (approximately 19 days) in the Séoune sub-basin, where the floods are voluminous. On the other hand, for the Tescou sub-basin, with rapid and small-volume floods, the time of transfer increases considerably $(8 \mathrm{~h})$ between the state of zero withdrawal (natural: 1.73 days) and the present state close to $6 \mathrm{Mm}^{3}$ (2.07 days). Concerning winter annual mean discharge in both sub-basins, the winter mean runoff coefficient decreased $31 \%$ and $\mathbf{4 2 \%}$, for the Séoune and Tescou sub-basins respectively, between the natural state and the present state. This reduction of the runoff coefficients does not correspond to the theoretical volumes stocked in the reservoirs. In other words, the theoretical volumes of the reservoirs cannot completely explain the observed strong reduction of the runoff coefficients. However, the consistency of the various controls, both on the observed time series and the modelled results, lend credibility to the overall results. These results should be taken into consideration in water management, if the climatic conditions and soil occupation don't change.

Key words: hydrological regime, use of water, irrigation, anthropogenic impact.

Les besoins en eau pour l'irrigation des cultures des sous-bassins du Tescou et de la Séoune (Bassin Adour-Garonne) se sont considérablement accrus ces trente dernières années. Cela s'est manifesté, entre autres, par la création de très nombreuses retenues collinaires. Pour le sous-bassin du Tescou, aux crues rapides et peu volumineuses, on totalise 184 retenues individuelles dont le volume théorique cumulé s'élève à $4,3 \mathrm{Mm}^{3}$ et qui interceptent environ un tiers $\left(92 \mathrm{~km}^{2}\right)$ de sa superficie $\left(287 \mathrm{~km}^{2}\right)$. Pour la Séoune, la pression due à l'usage de l'eau pour l'irrigation est un peu moins forte, compte tenu de sa taille $\left(463 \mathrm{~km}^{2}\right)$ et d'une ressource relativement abondante en hautes eaux. Près de 160 retenues collinaires sont dénombrées, elles représentent un volume de stockage théorique de $6,5 \mathrm{Mm}^{3}$ pour une superficie interceptée d'environ un quart du sous-bassin $\left(122 \mathrm{~km}^{2}\right)$. Le suivi hydrométrique des sous-bassins permet de disposer pour chacun d'eux d'une chronique de débit influencée $Q(t)$ sur une période d'observation d'environ 30 ans. Â partir des débits observés, notre objectif a été d'identifier et de quantifier l'impact 
anthropique pour trois composantes du régime hydrologique : crue, module et étiage. L'approche saisonnière des crues et modules a permis une différenciation de l'impact anthropique selon les saisons « hiver» (mois 12 à 6) et " été » (mois 7 à 11). Cette saisonnalisation hydrologique reflète bien le mode de gestion actuel de la ressource en eau de ces sous-bassins agricoles : stockage l'hiver et irrigation à partir des retenues collinaires durant les premiers mois d'été. Pour identifier l'impact, nous avons fait usage de tests de détection de rupture de stationnarité des chroniques hydrologiques : de LANG (2004) pour les crues et d'une adaptation du test de BoIS (1986) pour les modules et étiages des sous-bassins. Pour ce qui concerne les crues d'été et les modules d'été les tests montrent que les chroniques sont stationnaires sur l'ensemble de la période d'observation. Pour les crues et les modules d'hiver par contre, les tests relèvent des ruptures de stationnarité des chroniques qui vont nous permettre de définir des sous-périodes stationnaires. Ces souspériodes sont sensiblement cohérentes avec l'évolution des prélèvements théoriques cumulés de ces trente dernières années. L'analyse des ruptures de stationnarité menée sur les débits a de même été effectuée sur les pluies pour consolider nos résultats. La stationnarité des chroniques de pluie observées montre qu'il n'y a pas de composante climatique, autrement dit que la tendance détectée sur les débits est essentiellement d'origine anthropique. Il ne semble pas y avoir d'impact des éventuels pompages sur les débits d'étiage et tout particulièrement pour ce qui concerne la norme $V C N_{30}$ "débit moyen minimum annuel sur 30 jours consécutifs » des deux sous-bassins. Finalement, l'usage de l'eau pour l'irrigation affecte essentiellement les crues et les modules de la saison hiver. Un essai de quantification de cette tendance a été mené à partir d'une analyse statistique associant les observations des souspériodes stationnaires et les débits simulés pour une même référence pluviométrique. À partir des modélisations statistiques réalisées et des courbes de pression des besoins théoriques de l'irrigation, nous en avons déduit une tendance vraisemblable sur les quantiles de crue et les modules d'hiver. De manière générale, les crues de la saison hiver des deux sous-bassins sont fortement réduites en pointe et volume par l'ensemble des petites retenues. L'impact observé sur les quantiles de crue reste cohérent par rapport aux volumes théoriques prélevés dernièrement référencés. Les retenues collinaires sont sans action significative sur le temps de transfert (19 $j$ environ) du sous-bassin de la Séoune dont les crues sont volumineuses. Pour le Tescou par contre, aux crues rapides et peu volumineuses, le temps de transfert augmente sensiblement de $8 \mathrm{~h}$ entre l'état de prélèvement zéro (« naturel »: $1,73 \mathrm{j})$ et l'état actuel proche de $6 \mathrm{Mm}^{3}(2,07 \mathrm{j})$. Pour ce qui concerne les modules d'hiver des sous-bassins de la Séoune et du Tescou le coefficient d'écoulement moyen d'hiver diminue respectivement de $31 \%$ en moyenne et de $42 \%$ en moyenne entre l'état «naturel» et l'état anthropisé actuel. Cette diminution des coefficients d'écoulement est sans commune mesure avec les volumes théoriques stockés à partir des retenues collinaires. Autrement dit, les volumes théoriques des retenues collinaires ne peuvent expliquer à eux seuls la forte diminution des coefficients d'écoulement observés. Cependant, la cohérence des divers contrôles effectués, tant sur les chroniques observées que sur les modélisations effectuées, nous incitent à valider ces résultats. Ceux-ci devraient être retenus pour des objectifs de gestion immédiats ou ultérieurs de la ressource, si les conditions climatiques et d'occupation du sol n'évoluent guère.

Mots clés : régime hydrologique, usage de l'eau, irrigation, impact anthropique. 


\section{1 - INTRODUCTION}

Depuis une vingtaine d'années, l'irrigation soutenue des cultures dans le sud-ouest de la France a nécessité des besoins en eau de plus en plus importants. Cela a conduit à la création de très nombreuses retenues collinaires qui selon l'importance des volumes prélevés peuvent avoir un effet sensible sur le régime hydrologique des bassins versants. Celui-ci peut être décliné selon trois composantes: crues, modules et étiages. Tout naturellement, la question de l'impact des prélèvements sur la ressource disponible nous a été posée par divers acteurs de l'eau. Nous avons essayé d'y répondre à partir de l'exemple des sous-bassins du Tescou et de la Séoune pris dans le bassin AdourGaronne (VASQUEZ-PAULUS, 2004). Dans notre démarche, nous nous sommes intéressés à la détection de changements, dus à l'usage de l'eau pour l'irrigation, dans le comportement hydrologique des sous-bassins. Autrement dit, l'aspect non stationnaire des chroniques de débit observées a été pris en compte, comme cela est d'ailleurs la règle, pour traiter de la modélisation statistique des régimes hydrologiques des sous-bassins et par conséquent de l'impact des prélèvements d'eau pour l'irrigation. Par ailleurs, notre propos apporte quelques éléments de réflexion préalables à la classification des eaux courantes en états écologiques, objectif assigné aux états membres par la Directive Cadre Européenne (Directive 2000/60/CE). Cet objectif inclut l'impact des principaux usages de l'eau sur les régimes hydrologiques naturels des bassins versants.

\section{2 - CONTEXTE GÉNÉRAL DES SOUS-BASSINS DU TESCOU ET DE LA SÉOUNE}

\subsection{Cadre géographique et agricole des sous-bassins du Tescou et de la Séoune, données disponibles}

Le Tescou est un cours d'eau affluent en rive droite du Tarn, son bassin longiligne de nature principalement argileuse s'étend sur trois départements : Tarn, Haute-Garonne, Tarn-et-Garonne (figure 1). Le suivi hydrométrique est assuré par la DIREN Midi-Pyrénées à la station de St-Nauphary qui contrôle une superficie de $287 \mathrm{~km}^{2}$ (tableau 1). Le bassin très agricole présente un contraste marqué entre l'amont boisé ( $1 / 5$ de sa superficie) et l'aval où s'est développée la grande culture (maïs, tournesol...). Les besoins en eau aux cultures se sont considérablement accrus ces trente dernières années (CACG, 2001). On dénombre actuellement 184 retenues individuelles dont le volume théorique cumulé s'élève à plus de $4,3 \mathrm{Mm}^{3}$ (figure 2a) et qui interceptent environ un tiers $\left(92 \mathrm{~km}^{2}\right)$ de sa superficie. Les années 85 à 95 correspondent à une période de forte croissance des volumes stockés qui semble plus ou moins stabilisée depuis 1997. Par ailleurs deux retenues collectives (à hauteur de $1 \mathrm{Mm}^{3}$ ) et des arrosages directs par pompages en rivière (de juin à septembre) contribuent à l'irrigation des cultures (VASQUEZ-PAULUS, 2004). De ces différentes sources de prélèvement pour l'irrigation nous en déduisons la courbe cumulée des volumes théoriques prélevés (figure $2 b$ ) sur la saison hydrologique (mois 12 à 6) que nous appellerons ultérieurement « hiver ». 


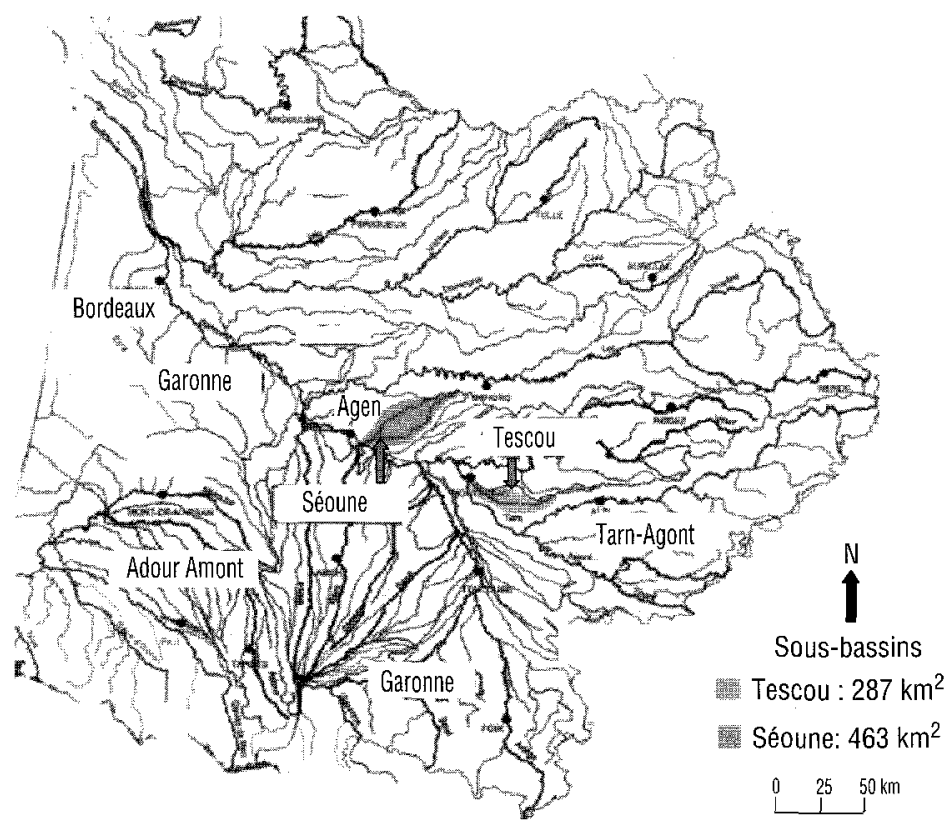

Figure 1 Situation des sous-bassins du Tescou et de la Séoune.

Geographical location of the Tescou and Séoune sub-basins.

Tableau 1 Données hydro-climatologiques disponibles.

Table 1 Available hydro-climatological data.

\begin{tabular}{cccc}
\hline Sous-bassin & $\begin{array}{c}\text { Débit } \\
\text { Chronique } Q(t) \text { et } \\
\text { Chronique journalière }\end{array}$ & Chronique journalière & Normales inter-décadaires \\
\hline Tescou & à St Nauphary & au poste de Salvagnac & Station météorologique d'Agen \\
$\left(287 \mathrm{~km}^{2}\right)$ & $1975-2003$ & $1968-2000$ & \\
Séoune & à St Pierre de Clairac & au poste de Laroque & \\
$\left(463 \mathrm{~km}^{2}\right)$ & $1969-2003$ & $1968-2000$ & \\
\hline
\end{tabular}

La Séoune conflue avec la Garonne en amont d'Agen. Son bassin versant de superficie $463 \mathrm{~km}^{2}$ à la station hydrométrique de St-Pierre-de-Clairac, gérée par la DIREN Aquitaine (tableau 1), est situé à la fois sur les départements du Lot-et-Garonne, du Tarn-et-Garonne et du Lot (figure 1). Au voisinage de la plaine de la Garonne se situent des cultures d'été (maïs, tournesol...), plus au nord le plateau de calcaire blanc perméable (haut Quercy-Blanc) est à cultures essentiellement céréalières et protéagineuses. De nombreuses retenues collinaires ont été implantées, 160 retenues qui représentent un volume de stockage de $6,5 \mathrm{Mm}^{3}$ (figure 2c) et environ un quart du bassin versant capté $\left(122 \mathrm{~km}^{2}\right)$. Une forte poussée est observée au cours des années 1985-1991 et semble depuis stabilisée (DELBREILH, 1993). 
Sous-bassin du Tescou : courbe cumulée des prélèvements par retenues collinaires individuelles

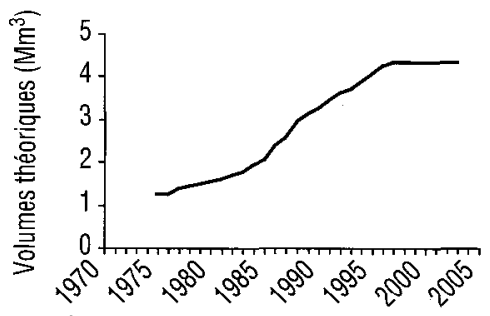

(a)
Sous-bassin du Tescou : courbe cumulée des prélèvements sur la saison hydrologique " hiver :

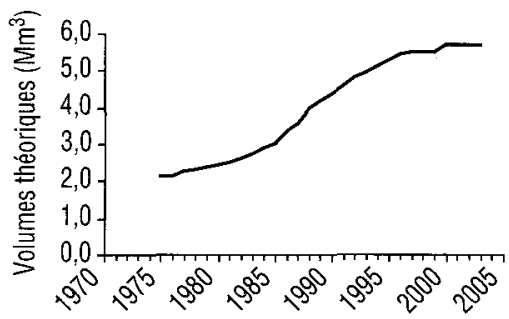

(b)

Sous-bassin de la Séoune : courbe cumulée des prélèvements par retenues collinaires individuelles

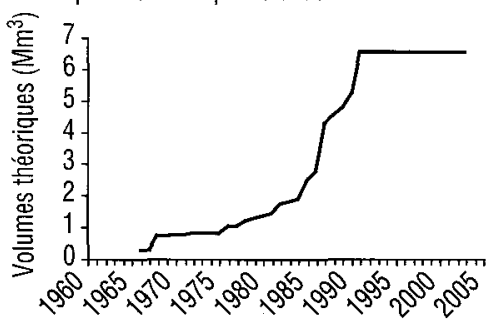

(c)

Figure 2 Volumes théoriques prélevés sur la saison hydrologique « hiver ».

Theoretical volumes withdrawn in the winter season.

\subsection{Typologie des crues et saisonnalisation hydrologique}

La typologie des crues des deux sous-bassins est bien différenciée. La séquence $Q(t)$ présentée ci-après (figure 3 ) montre que les crues du sous-bassin du Tescou sont beaucoup plus rapides et peu soutenues par rapport aux crues complexes et volumineuses de la Séoune.

Pour les deux sous-bassins le caractère saisonnier des crues est bien marqué. L'échantillonnage des plus fortes crues au dessus d'un seuil des chroniques $Q(t)$ montre que les crues du Tescou et de la Séoune, respectivement 60 et 70 crues, se répartissent pour la majorité d'entre elles du mois de décembre au mois de juin (figure 4).

Ceci nous permet de considérer deux saisons hydrologiques : une saison " hiver » (mois 12 à 6) et une saison " été » (mois 7 à 11). Pour la suite de notre propos, les termes hiver et été seront simplement utilisés. Cette saisonnalisation est cohérente avec l'usage de l'eau. Les réserves sont constituées durant l'hiver à partir des retenues collinaires (+ arrosage direct en juin) et sont utilisées l'été pour l'irrigation des cultures qui en principe porte sur 4 mois, de juin à septembre. Cette saisonnalisation va nous permettre de différencier l'impact des prélèvements sur les régimes hydrologiques des sous-bassins selon qu'il s'agit de stockage l'hiver à partir des retenues collinaires (+ éventuels pompages directs en juin) et d'irrigation d'appoint l'été à partir d'éventuels pompages directs en rivière. 


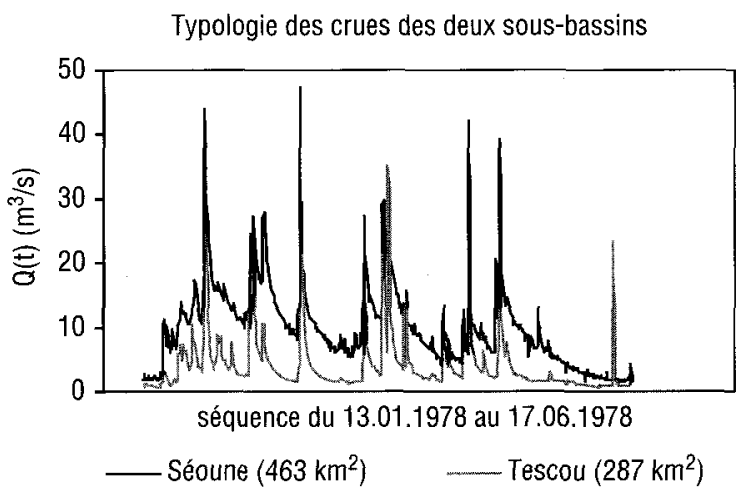

Figure 3 Aperçu sur la typologie des crues des deux sous-bassins.

Overview of flood typology of the sub-basins.

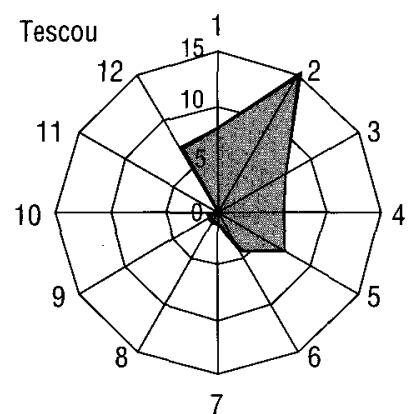

Seuil $=20,5 \mathrm{~m}^{3} / \mathrm{s}$

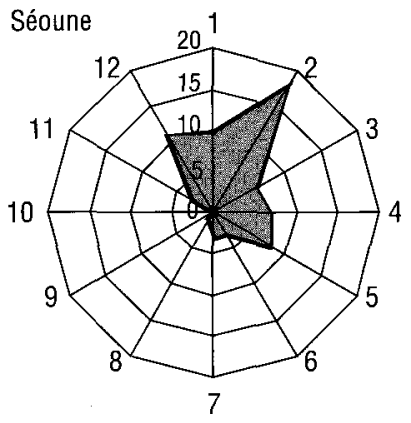

Seuil $=17,9 \mathrm{~m}^{3} / \mathrm{s}$

Figure 4 Saisonnalité des crues.

Seasonal occurrence of floods.

\section{3 - IDENTIFICATION ET MODÉLISATION DE L'IMPACT DES PRÉLĖVEMENTS SUR LES CRUES D'HIVER ET D'ÉTÉ DE LA SÉOUNE ET DU TESCOU}

\subsection{Identification de sous-périodes stationnaires pour les crues d'hiver et d'été}

Le test mis en œuvre pour la détection de ruptures de stationnarité d'une chronique hydrologique $Q(t)$ est le test de LANG et al. (2004) dont nous donnons ci-après un bref rappel. 


\subsubsection{Rappel théorique sur le test de LANG (1995)}

Ce test initié par LANG (1995) considère que le processus d'occurrence homogène des crues supérieures à un seuil suit une loi de Poisson de paramètre $\mu$ (nombre moyen d'événements par an). La probabilité d'observer $k$ crues sur la période $[0, t]$ est :

$$
\operatorname{Prob}\left[m_{t}=k \operatorname{sur}[0, t]\right]=w_{k}(t)=e^{-\mu t} \frac{(\mu t)^{k}}{k !}
$$

où $\quad m_{t}$ est le nombre d'événements supérieurs au seuil sur $[0, t]$

avec $E\left[m_{t}\right]=\mu t$ et $\sigma^{2}\left[m_{t}\right]=\mu t ; l_{t}=\frac{\sigma^{2}}{E}=1$ (indice de dispersion)

Et donc, conditionnellement à l'observation de $n$ crues sur $\left[0, t_{\text {fin }}\right]$ il s'ensuit la probabilité $w_{k}^{*}(t)$ d'observer $k$ crues sur $[0, t]$ :

$$
w_{k}^{*}(t)=C_{n}^{k}\left(\frac{t}{t_{\text {fin }}}\right)^{k}\left(1-\frac{t}{t_{\text {fin }}}\right)^{n-k}
$$

Les bornes de l'intervalle de tolérance au seuil de confiance $\alpha$ de la variable $m_{t}$ est une ellipse symétrique de grand axe $\mu t$ et sont définies par résolution numérique de l'équation (2) pour la probabilité $\alpha / 2 \%$ et $1-\alpha / 2 \%$ (LANG et al., 1999).

Nous en donnons une illustration ci-après, pour tester la stationnarité des chroniques de crue d'hiver ou d'été des sous-bassins de la Séoune et du Tescou.

\subsubsection{Crues d'hiver des sous-bassins de la Séoune et du Tescou}

Pour des pas de temps $t$ de calcul régulièrement espacés, il est possible de représenter le report chronologique du numéro de la crue en fonction de sa date d'occurrence $M \exp (t)$ ainsi que l'intervalle de tolérance à $90 \%$ [M5 \%; M95\%] si l'on suppose que le processus de ces événements est poissonien (figure 5). Le processus est dit stationnaire selon le test de LANG (1995) si la courbe $M \exp (t)$ est située à l'intérieur de l'intervalle de tolérance à $90 \%$ [M5 \%; M95 \%]. Ce test a été récemment recadré (LANG et al., 2004) pour passer d'une probabilité locale à une date donnée à une règle de décision pour un point quelconque de la chronique. II en résulte qu'une chronique n'est pas stationnaire si plus de $29 \%$ des points sont en dehors de l'intervalle de contrôle à $90 \%$ (26\% et $21 \%$ respectivement pour des I.C. à $95 \%$ et $99 \%$ ). Ce seuil a été déterminé par simulation de Monte-Carlo. La chronique des débits de crue du sous-bassin de la Séoune n'est pas stationnaire sur la période 1968-2003, nous en donnons une illustration pour les débits de pointe (figure 5). Le test nous permet par approches successives d'identifier deux sous-périodes stationnaires $1968-1988$ et $1989-2003$.

De même, pour le sous-bassin du Tescou la chronique des débits de pointe n'est pas stationnaire sur la période 1975-2003. Quatre sous-périodes stationnaires peuvent être identifiées (figure 6). 
Débit de pointe des crues d'hiver de la Séoune : 1968-2003

Choix du seuil $=20,2 \mathrm{~m}^{3} / \mathrm{s}, \mathrm{Mu}=2,02$

Non-stationnarité du processus d'occurrence de Poisson

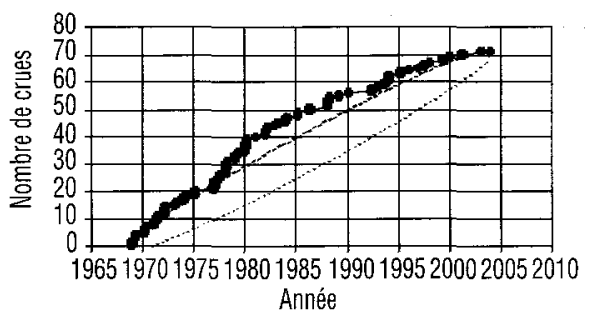

...M95\% $\rightarrow$ Mexp ....... M5\%

Débit de pointe des crues d'hiver de la Séoune : 1968-1988

Choix du seuil $=17,4 \mathrm{~m}^{3} / \mathrm{s}, \mathrm{Mu}=2,03$

Stationnarité du processus d'occurrence de Poisson

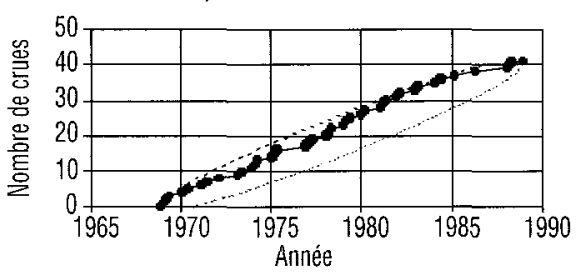

.... M95\% ๑-Mexp .......M5\%
Débit de pointe des crues d'hiver de la Séoune : 1989-2003

Choix du seuil $=13 \mathrm{~m}^{3} / \mathrm{s}, \mathrm{Mu}=2,06$

Stationnarité du processus d'occurrence de Poisson

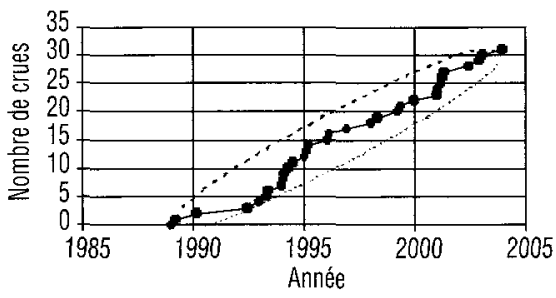

.... M95\% $\rightarrow$ Mexp ....... M5\%

Figure 5 Séoune - Chronique des débits de pointe d'hiver non stationnaire, identification des 2 sous-périodes stationnaires.

Séoune - non-stationary winter peak flood time series: identification of 2 stationary sub-periods. Choix du seuil $=18,2 \mathrm{~m} / \mathrm{s}, M U=2,3$

Non-stationnarité du processus d'occurrence de Poisson

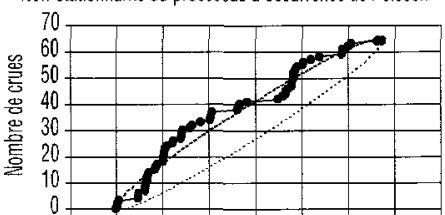

197019751980198519901995200020052010 Année

$\ldots . . M 95 \% \rightarrow$ Mexp $\quad \ldots \ldots . . . M 5 \%$

$1975-1985$

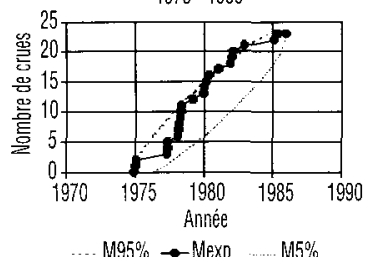

$1992-1996$

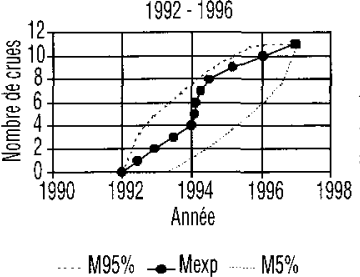

$1986-1991$

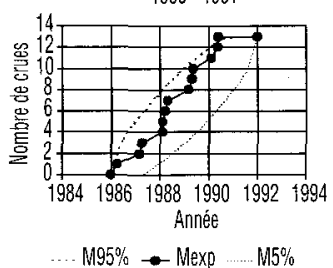

$1997-2003$

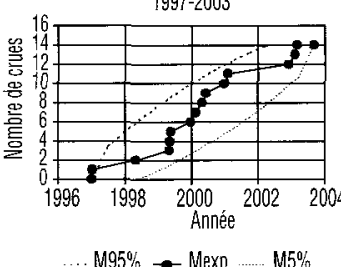

Figure 6 Tescou - Chronique des débits de pointe d'hiver non stationnaire, identification des 4 sous-périodes stationnaires.

Tescou - non-stationary winter peak flood time series: identification of 4 stationary sub-periods. 


\subsubsection{Crues d'été des sous-bassins de la Séoune et du Tescou}

Nous rappelons que les crues d'été concernent la saison hydrologique des mois de juillet à novembre. Pour les deux sous-bassins les crues d'été sont rapides et peu soutenues (ct. modélisation ultérieure). Pour les crues d'été et à titre d'exemple nous montrons que le processus des crues est poissonien en vérifiant que le ratio $(R)$ de Poisson ou encore indice de dispersion est sinon égal à 1 du moins compris dans son intervalle de confiance à $90 \%$ pour le choix du seuil effectué. Que ce soit pour le sous-bassin de la Séoune ou du Tescou les chroniques des crues d'été retenues ne montrent pas de ruptures de stationnarité tant pour les débits de pointe que pour les débits de crue moyens de $12 \mathrm{~h}$ (figures 7 et 8 ).

Débit de pointe des crues d'été du sous-bassin de la Séoune respect du ratio $R=1$ de la loi de Poisson

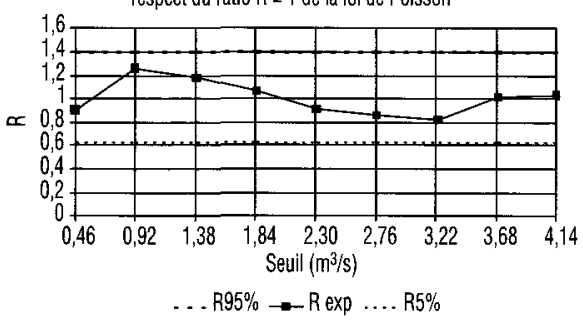

Débit moyen sur 12 h des crues d'été du sous-bassin de la Séoune respect du ratio $R=1$ de la loi de Poisson

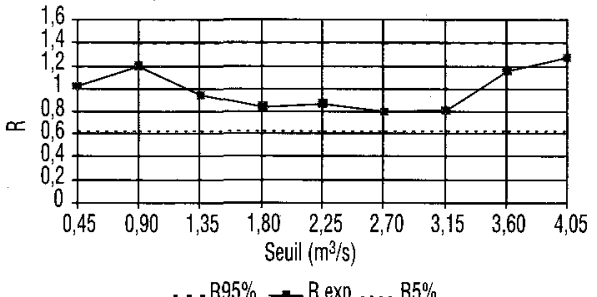

Débit de pointe des crues d'étê du sous-bassin de la Séoune Choix du seuil $=2,30 \mathrm{~m}^{3} / \mathrm{s}, \mathrm{Mu}=2$

Stationnarité du processus d'occurrence de Poisson

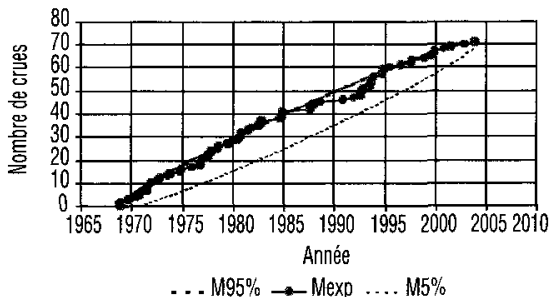

Débit moyen sur $12 \mathrm{~h}$ des crues d'été du sous-bassin de la Séoune Choix du seuil $=2,25 \mathrm{~m}^{3} / \mathrm{s}, \mathrm{Mu}=1,73$

Stationnarité du processus d'occurrence de Poisson

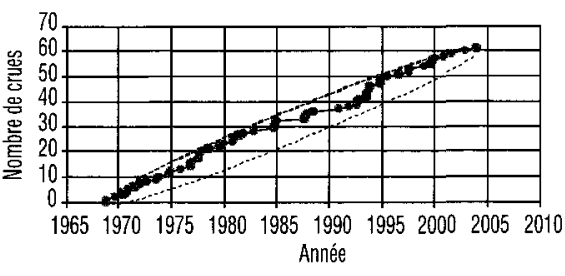

. . M95\% $\rightarrow-M \operatorname{exp~...M5\% ~}$

Figure 7 Stationnarité des chroniques de crue d'été du sous-bassin de la Séoune.

Stationnarity of summer flood time series for the Séoune sub-basin. 
Débit de pointe des crues d'été du sous-bassin du Tescou respect du ratio $R=1$ de la loi de Poisson

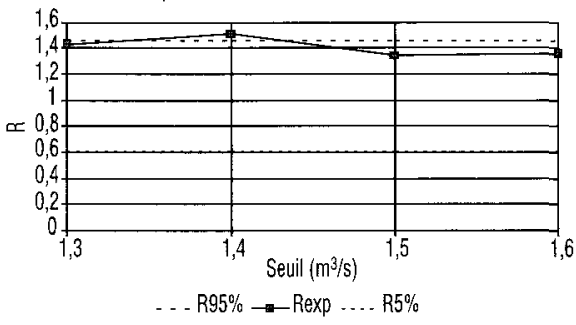

Débit moyen sur $12 \mathrm{~h}$ des crues d'été du sous-bassin du Tescol respect du ratio $\mathrm{R}=1$ de la loi de Poisson

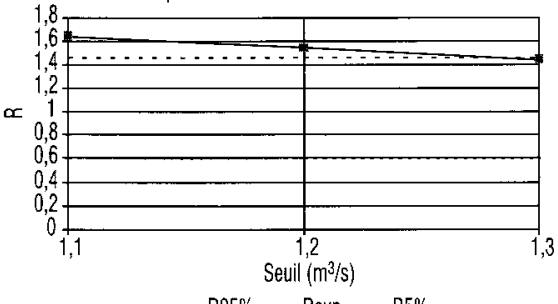

$\ldots R 95 \% \rightarrow-\operatorname{Rexp} \ldots . . . R 5 \%$
Débit de pointe des crues d'été du sous-bassin du Tescou

Choix du seuit $=1,5 \mathrm{~m}^{3} / \mathrm{s}, \mathrm{MU}=1,74$

Stationnarité du processus d'occurrence de Poisson

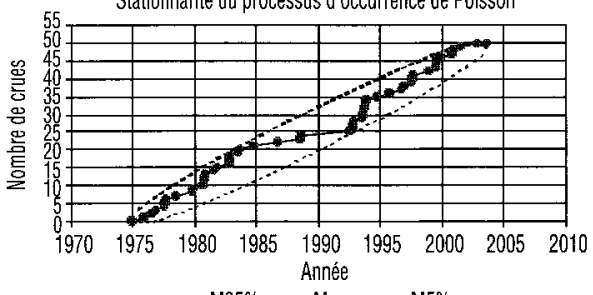

- . - M95\% - Mexp .... M5\%

Débit moyen sur $12 \mathrm{~h}$ des crues d'été du sous-bassin du Tescou Choix du seuil $=1,3 \mathrm{~m}^{3} / \mathrm{s}, \mathrm{Mu}=1,62$

Stationnarité du processus d'occurrence de Poisson

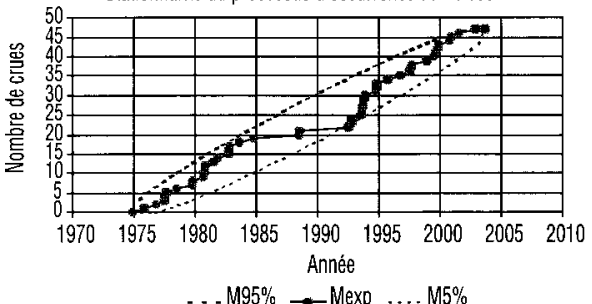

... M95\% _.Mexp ....M5\%

Figure 8 Stationnarité des chroniques de crue d'été du sous-bassin du Tescou.

Stationnarity of summer flood time series for the Tescou sub-basin.

\subsubsection{Vérification de la stationnarité des pluies maximales d'hiver et d'été}

D'une manière générale, pour considérer que les résultats précédemment obtenus sont essentiellement dus au mode d'usage de l'eau pour l'irrigation il est nécessaire de vérifier que les pluies maximales à l'origine des crues d'hiver et d'été sont stationnaires sur la période d'observation des débits. À partir des chroniques de pluie disponibles (tableau 1) nous avons réalisé un échantillonnage sup-seuil saisonnalisé des pluies maximales de durée compatible avec la dynamique des crues des sous-bassins. Ainsi, pour les crues d'été rapides des deux sous-bassins nous avons retenu les pluies maximales de $24 \mathrm{~h}$ (figure 9). Pour les crues d'hiver de grande inertie de la Séoune et relativement rapides du Tescou, nous avons respectivement retenu les pluies maximales de 5 jours et de $24 \mathrm{~h}$ (figure 10). 


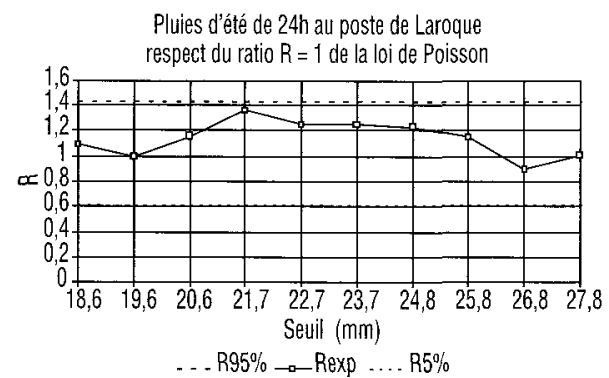

Pluies d'été de 24 h au poste de Salvagnac respect du ratio $R=1$ de la loi de Poisson

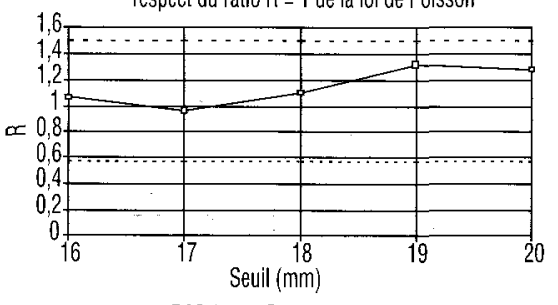

. . . R95\% $\rightarrow$ - Rexp .... R $5 \%$
Pluies d'été de 24h au poste de Laroque

Choix du seuil $=20,6 \mathrm{~mm}, \mathrm{Mu}=2,2$ Stationnarité du processus d'occurrence de Poisson

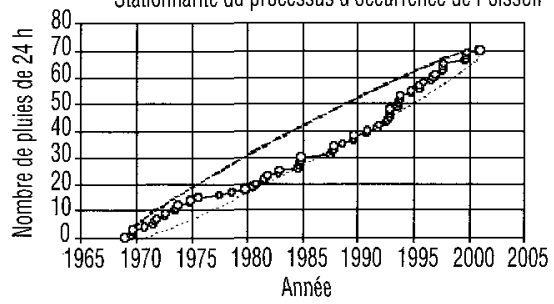

... M95\% $\rightarrow$ - Mexp .... M5\%

Pluies d'été de 24h au poste de Salvagnac

Choix du seuil $=17 \mathrm{~mm}, \mathrm{Mu}=3$

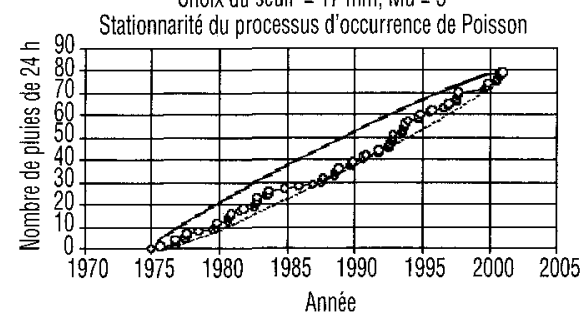

Figure 9 Stationnarité des pluies maximales d'été.

Stationnarity of maximum summer rainfalls.

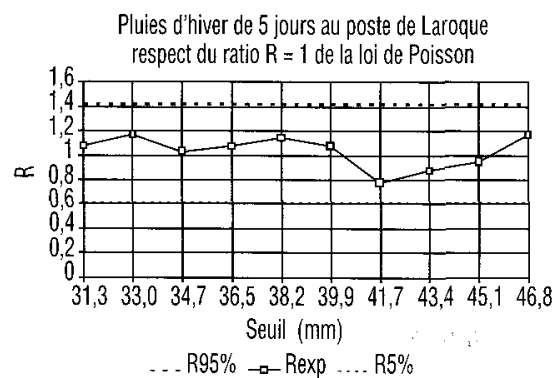

Pluie d'hiver de 24 h au poste de Salvagnac respect du ratio $\mathrm{R}=1$ de la loi de Poisson

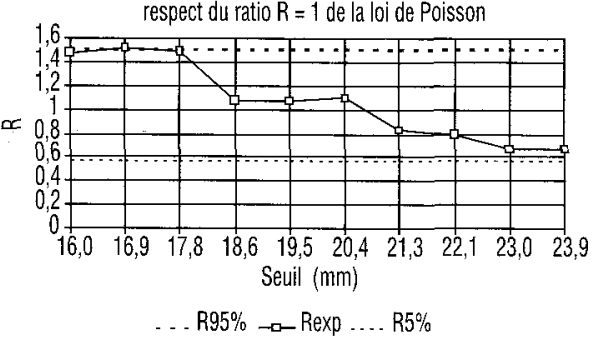

Pluies d'hiver de 5 jours au poste de Laroque Choix du seuil $=43,4 \mathrm{~mm}, \mathrm{Mu}=2,1$

Stationnarité du processus d'occurrence de Poisson

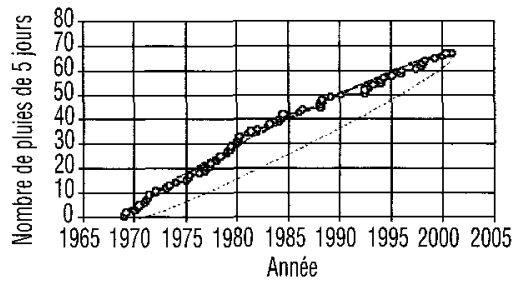

_. M95\% _. Mexp ....M5\%

Pluies d'hiver de $24 \mathrm{~h}$ au poste de Salvagnac Choix du seuil $=22.1 \mathrm{~mm}, \mathrm{Mu}=2$

Stationnarité du processus d'occurrence de Poisson

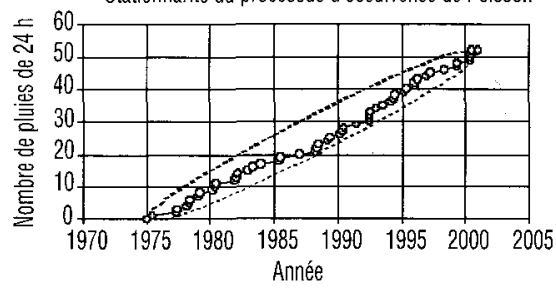

... M95\% $\rightarrow$ - Mexp .... M5\%

Figure 10 Stationnarité des pluies maximales d'hiver.

Stationnarity of maximum winter rainfalls. 


\subsubsection{Conclusion partielle}

Le fait de ne pas avoir constaté d'impact sur les crues d'été pour les deux sous-bassins nous permet de dire que la ressource nécessaire à l'irrigation durant la saison été (mois 7 à 11) dépend essentiellement du volume d'eau stocké par les retenues collinaires pendant la saison hiver (mois 12 à 6). Autrement dit, les éventuels pompages pendant la période d'irrigation n'influencent pas les crues de la saison été. Par contre, pour les crues de la saison hiver l'intensification des prélèvements, pour l'essentiel à partir des retenues collinaires durant ces trente dernières années environ, a influencé les chroniques de débit $Q(t)$ observées pour lesquelles nous avons identifié des ruptures de stationnarité. Ces ruptures de stationnarité mises en évidence sur les débits de crue d'hiver sont essentiellement dues au mode d'usage de l'eau pour l'irrigation, il n'y a pas d'incidence climatique. Nous avons en effet vérifié le caractère stationnaire des pluies maximales, quelle que soit d'ailleurs la saison considérée, aux postes de Laroque (1969-2000) et de Salvagnac (1975-2000).

\subsection{Modélisation statistique des régimes de crue d'été de la Séoune et du Tescou}

Nous avons montré au paragraphe précédent la stationnarité des crues d'été observées. À toutes fins utiles, nous en donnons ci-après une formulation analytique du régime selon le concept $\mathrm{QdF}$.

\subsubsection{Bref rappel sur la modélisation débit-durée-fréquence ( $\mathrm{QdF})$}

Les variables hydrologiques étudiées concernent le débit moyen $V d$ sur une durée continue $d$ et le débit seuil $Q d$ continûment dépassé sur la durée $d$, maximaux sur l'épisode de crue. Ces variables sont égales au débit de pointe QIX pour $d=0$. Les durées $d$ retenues doivent être compatibles avec la dynamique de crue des sous-bassins. La modélisation statistique des variables supérieures à un seuil (LANG et al., 1999) est réalisée d'après le modèle QdF convergent de JAVELLE et al. (2000). Ce modèle repose sur une propriété observée des distributions expérimentales à converger vers les faibles périodes de retour et sur une propriété d'affinité orthogonale des distributions théoriques relatives au différentes durées $d$ choisies (JAVELLE, 2001). Ces propriétés induisent une forme analytique à faible nombre de paramètres calés sur l'ensemble des échantillons de durée $d$ constitués et dont le concept est indépendant de la loi de probabilité théorique choisie $V(0, T)$. Nous présentons ci-après les modèles $\mathrm{QdF}$ établis. Nous noterons que les durées $\Delta$ des régimes de crue d'été observés caractérisent des temps de transfert rapides des écoulements. Les volumes mis en jeu sont relativement peu importants, comparativement aux crues d'hiver des sous-bassins.

\subsubsection{Régime des crues d'été du sous-bassin de la Séoune}

La loi de probabilité théorique $V(0, T)$ choisie pour représenter les échantillons $V_{d}$ de la saison été est la loi de Pareto. Les paramètres $a(0), x_{0}(0)$ et $k$ sont les paramètres de la distribution théorique des débits de pointe obtenus par modélisation QdF (4) et ajustés par la méthode des moments pondérés (HOSKING et al., 1985). 


$$
V(d, T)=\frac{x_{0}(0)+\frac{a(0)}{k}\left[1-\frac{1}{(\mu T)^{k}}\right]}{1+d / \Delta}
$$

où :

$\mu$ : nombre moyen d'événements retenus par an

$a(0)$ : paramètre d'échelle

$x_{0}(0)$ : paramètre de position

$k$ : coefficient de forme

$\Delta$ : est équivalent à un temps et renseigne sur la forme des crues. Ce paramètre permet de passer de la distribution consolidée des débits de pointe aux distributions de durées $d$ compatibles avec la dynamique de crue.

On en déduit l'expression (5) :

$$
V(d, T)=\frac{4,55+\frac{2,03}{-0,53}\left[1-\frac{1}{(1,6 T)^{-0,53}}\right]}{1+d / 0,73}
$$

Les quantiles centrés déduits de (5) sont présentés au tableau 2 ci-après avec leur intervalle de confiance à $70 \%$ :

Nous rappelons qu'à ces quantiles de débit moyen (5) peuvent être associés selon (6) des quantiles de débit seuil (7) qui vont permettre de construire des hydrogrammes de projet (GALÉA et PRUDHOMME 1994 ; JAVELLE, 2001).

$$
\begin{gathered}
Q(d, T)=\frac{\partial(d V(d, T))}{\partial d} \\
Q(d, T)=\frac{4,55+\frac{2,03}{-0,53}\left[1-\frac{1}{(1,6 T)^{-0,53}}\right]}{(1+d / 0,73)^{2}}
\end{gathered}
$$

\subsubsection{Régime des crues d'été du sous-bassin du Tescou}

Un modèle QdF privilégiant la loi exponentielle a été calé (8) à partir d'échantillons sup-seuil $(\mu=1,72)$ extraits de la chronique observée $Q(t)$ stationnaire de 29 années (1975-2003). Les quantiles centrés relatifs aux différentes durées et périodes moyennes de retour ainsi que l'intervalle de confiance à $70 \%$ sont donnés au tableau 3 à toutes fins utiles.

$$
V(d, T)=\frac{5 \ln (T)+4,8}{1+d / 1,01}
$$


Tableau 2 Quantiles des crues d'été du sous-bassin de la Séoune.

Table 2 Summer flood quantiles for the Séoune sub-basin.

\begin{tabular}{|c|c|c|c|c|c|c|c|c|c|c|c|c|}
\hline \multirow{4}{*}{$\begin{array}{c}T \\
\text { (an) }\end{array}$} & \multicolumn{12}{|c|}{ Quantiles $V(d, T)$ des crues d'été de la Séoune $\left(\mathrm{m}^{3} / \mathrm{s}\right)$} \\
\hline & \multicolumn{12}{|c|}{$d(j)$} \\
\hline & \multicolumn{3}{|c|}{0} & \multicolumn{3}{|c|}{0,5} & \multicolumn{3}{|c|}{1} & \multicolumn{3}{|c|}{2} \\
\hline & $15 \%$ & $\begin{array}{c}\text { Valeur } \\
\text { centrée }\end{array}$ & $85 \%$ & $15 \%$ & $\begin{array}{l}\text { Valeur } \\
\text { centrée }\end{array}$ & $85 \%$ & $15 \%$ & $\begin{array}{c}\text { Valeur } \\
\text { centrée }\end{array}$ & $85 \%$ & $15 \%$ & $\begin{array}{l}\text { Valeur } \\
\text { centrée }\end{array}$ & $85 \%$ \\
\hline 1 & 5,16 & 5,6 & 6,0 & 3,0 & 3,3 & 3,6 & 2,2 & 2,4 & 2,6 & 1,4 & 1,5 & 1,6 \\
\hline 2 & 7,0 & 7,8 & 8,7 & 4,1 & 4,6 & 5,1 & 2,9 & 3,3 & 3,7 & 1,9 & 2,1 & 2,3 \\
\hline 5 & 10,3 & 12,2 & 14,1 & 6,2 & 7,3 & 8,4 & 4,4 & 5,2 & 6,0 & 2,8 & 3,3 & 3,8 \\
\hline 10 & 13,4 & 17,4 & 21,4 & 7,9 & 10,3 & 12,7 & 5,6 & 7,3 & 9,0 & 3,5 & 4,6 & 5,7 \\
\hline
\end{tabular}

Tableau 3 Quantiles des crues d'été du sous-bassin du Tescou.

Table 3 Summer flood quantiles for the Tescou sub-basin.

\begin{tabular}{|c|c|c|c|c|c|c|c|c|c|c|c|c|c|c|c|c|c|c|}
\hline \multirow{4}{*}{$\begin{array}{c}T \\
\text { (an) }\end{array}$} & \multicolumn{18}{|c|}{ Quantiles V $(\mathrm{d}, \mathrm{T})$ des crues d'été du Tescou $\left(\mathrm{m}^{3} / \mathrm{s}\right)$} \\
\hline & \multicolumn{18}{|c|}{$d(j)$} \\
\hline & & 0 & & & 0,5 & & & 1 & & & 1,5 & & & 2 & & & 3 & \\
\hline & $15 \%$ & $\begin{array}{l}\text { Valeur } \\
\text { centrée }\end{array}$ & & $15 \%$ & $\begin{array}{l}\text { Valeur } \\
\text { centrée }\end{array}$ & $85 \%$ & & $\begin{array}{l}\text { Valeur } \\
\text { centrée }\end{array}$ & $85 \%$ & $15 \%$ & $\begin{array}{l}\text { Valeur } \\
\text { centrée }\end{array}$ & $85 \%$ & & $\begin{array}{l}\text { Valeur } \\
\text { centrée }\end{array}$ & $85 \%$ & $15 \%$ & $\begin{array}{l}\text { Valeur } \\
\text { centrée }\end{array}$ & $85 \%$ \\
\hline 1 & 4,1 & 4,8 & 5,6 & 2,7 & 3,2 & 3,7 & 2,1 & 2,4 & 2,8 & 1,6 & 1,9 & 2,2 & 1,4 & 1,6 & 1,9 & 1,0 & 1,2 & 1,4 \\
\hline 2 & 7,8 & 8,3 & 8.9 & 5,2 & 5,6 & 5,9 & 3,9 & 4,2 & 4,5 & 3,1 & 3,3 & 3,6 & 2,6 & 2,8 & 3,0 & 2,0 & 2,1 & 2,2 \\
\hline 5 & 11,6 & 12,9 & 14,2 & 7,8 & 8,6 & 9,5 & 5,8 & 6,5 & 7,1 & 4,7 & 5,2 & 5,7 & 3,9 & 4,3 & 4,8 & 2,9 & 3,3 & 3,6 \\
\hline 101 & 14,4 & 16,4 & 18,3 & 9,7 & 11,0 & 12,3 & 7,3 & 8,2 & 9,2 & 5,8 & 6,6 & 7,4 & 4,8 & 5,5 & 6,2 & 3,6 & 4,1 & 4,6 \\
\hline
\end{tabular}

\subsection{Quantification de l'impact des retenues collinaires sur les régimes de crue d'hiver du Tescou et de la Séoune}

\subsubsection{Présentation de la démarche}

Le régime des crues d'hiver des sous-bassins évolue dans le temps sous la pression croissante des prélèvements effectués pour l'irrigation. Cette évolution a été détectée à partir d'un test de rupture de stationnarité du processus d'occurrence des crues (LANG et al., 2004) appliqué aux chroniques observées $Q(t)$ et qui a permis d'identifier des sous-périodes stationnaires. Notre objectif est de modéliser à partir de ces sous-périodes stationnaires (4 pour le Tescou et 2 pour la Séoune) et des volumes théoriques prélevés (figure 2) la tendance des régimes de crue associés et d'en déduire, selon toute vraisemblance, une estimation du « régime naturel » (pression de prélèvement nulle). Nous pourrons ensuite proposer une réponse à la question récurrente de l'impact des différents états d'anthropisation des deux sous-bassins sur les quantiles de crue « naturels ». Notre démarche méthodologique est présentée ci-après pour les crues d'hiver du sous-bassin du Tescou. 


\subsubsection{Modélisation statistique des régimes de crue d'hiver du sous-bassin du Tescou}

Dans notre démarche sont associées conjointement les modélisations QdF relatives aux chroniques $Q(t)$ et aux chroniques de débit quotidien $E_{i}$ représentatives du niveau d'anthropisation des quatre sous-périodes stationnaires identifiées. Les chroniques de débit quotidien $E_{i}$ font référence à une même pluie d'entrée (1975-2000) contrairement aux chroniques $Q(t)$ de chaque souspériode (1975-1985, 1986-1991, 1992-1996, 1997-2003). Les chroniques $E_{i}$ (tableau 4) sont obtenues par extension des chroniques de débit quotidien observées de chaque sous-période stationnaire par le modèle pluie-débit GR4J (PERRIN, 2002). Autrement dit, GR4J nous permet d'établir sur la durée d'observation de la pluie (1975-2000) une chronique de débit quotidien stationnaire pour chacune des sous-périodes (ex. : figure 11).

Tableau 4 Modélisation QdF des crues d'hiver du sous-bassin du Tescou.

Table 4 QdF Modeling of winter floods for the Tescou sub-basin.

\begin{tabular}{|c|c|c|c|c|c|c|}
\hline \multicolumn{7}{|c|}{ Modélisation des crues $V(d, T)$ du sous-bassin du Tescou sur la saison hiver $\left(\mathrm{m}^{3} / \mathrm{s}\right)$} \\
\hline \multicolumn{4}{|c|}{ Chronique a(t) observée (1975-2003) } & \multicolumn{3}{|c|}{ Chroniques quotidiennes $E_{i}(1975-2000)$} \\
\hline $\begin{array}{l}\text { Sous-périodes } \\
\text { stationnaires }\end{array}$ & \multicolumn{3}{|c|}{ Paramètres $Q d F$} & $\begin{array}{c}\text { Sous-période stationnaire } \\
+(\text { simulation GR4I })\end{array}$ & \multicolumn{2}{|c|}{ Paramètres QdF } \\
\hline 1975-1985 & 13,0 & 38,7 & 1,89 & $E_{1}=1975-1985+(1986-2000)$ & 18,62 & $41,11 \quad 1,89$ \\
\hline $1986-1991$ & 10,09 & 15,21 & 2,54 & $975-1985)+1986-1991+(1992-2000)$ & 10,80 & $19,39 \quad 1,99$ \\
\hline 1992-1996 & 12,65 & 37,79 & 1,93 & $E_{3}=(1975-1991)+1992-1996+(1997-2000)$ & 14,05 & $25,51 \quad 1,81$ \\
\hline 1997-2003 & 8,78 & 16,01 & 2,38 & $E_{4}=(1975-1996)+1997-2000$ & 11,66 & $20,26 \quad 2,2$ \\
\hline
\end{tabular}

Débits de crue d'hiver de $24 \mathrm{~h}$ du sous-bassin du Tescou Chronique observée 1975-2003: choix du seuil $=13,9 \mathrm{~m}^{3} / \mathrm{s}, \mathrm{Mu}=2$

Non stationnarité du processus d'occurrence de Poisson

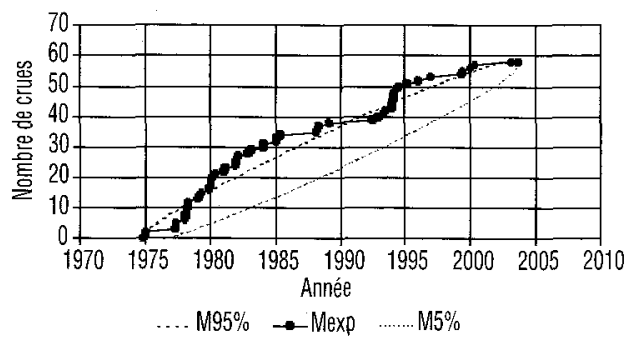

Débits de crue d'hiver de 24h du sous-bassin du Tescou

Chronique E1 : choix du seuil $=14,5 \mathrm{~m}^{3} / \mathrm{s}, \mathrm{Mu}=2,3$

Stationnarité du processus d'occurrence de Poisson

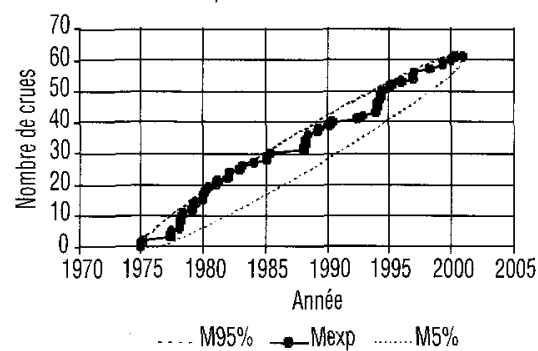

Figure 11 Non-stationnarité et stationnarité des crues de $24 \mathrm{~h}$ des chroniques respectives $\mathrm{Q}(\mathrm{t})$ et $\mathrm{Ei}$ (ex. : $\mathrm{E} 1$ ).

Non-stationnarity and stationnarity of 24- $h$ winter flood for the $Q(t)$ and Ei (ex. : E1) time series respectively. 
Pour les chroniques $Q(t)$ et $E_{i}$ la modélisation $Q d F$ à trois paramètres privilégie la loi exponentielle (ex. : figure 12 ; tableau 4).

Pour les chroniques $E_{i}$ de débit journalier nous avons calé un modèle $Q d F$ dont les paramètres de la distribution théorique des débits de $24 \mathrm{~h}\left(\mathrm{a}(1), x_{0}(1)\right)$ sont consolidés par modélisation sur l'ensemble de l'information échantillonnée relative aux pas de temps $d$ (de 1 j à 4 j). Ceci peut se faire en vertu de la liberté de choix de la durée $d$ de référence qu'offre le concept de modélisation (JAVELLE 2001). Pour ce qui concerne les quantiles de débit de pointe QIX (ex. : figure 12), les paramètres de la loi $a(0)$ et $x_{0}(0)$ se déduisent par pondération (ratio : 1,5) des paramètres de la distribution théorique des débits de $24 \mathrm{~h}$, soit $a(0)=a(1)^{*} 1,5$ et $x_{0}(0)=a(1)^{*} 1,5$. Le ratio 1,5 est le rapport entre le quantile annual de pointe et le quantile annual de $24 \mathrm{~h}$. Ce coefficient s'est révélé pratiquement constant lors des 4 modélisations $Q d F$ des chroniques $Q(t)$ observées. Pour ce qui concerne la durée caractéristique de crue $\Delta$, celle-ci est déduite des échantillons observés de durées d retenues (1 j à 4 j) et théorique pour ce qui concerne les débits de pointe QIX (JAVELLE et al., 1999).

L'exploitation des deux types de chroniques permet une consolidation réciproque des jeux de paramètres obtenus. En effet, bien que le processus d'occurrence des pluies maximales ait été montré stationnaire nous ne pouvions pas nous satisfaire des seules modélisations $Q d F$ réalisées sur les chroniques $Q(t)$ des 4 sous-périodes stationnaires. II était nécessaire de les consolider par une analyse statistique des débits pour une même référence pluviométrique (1975-2000).

Bassin du Tescou à St-Nauphary $\left(S=287 \mathrm{~km}^{2}\right)$ Modèle QdF sur la période Q(t) observée : saison hiver 1975-1985

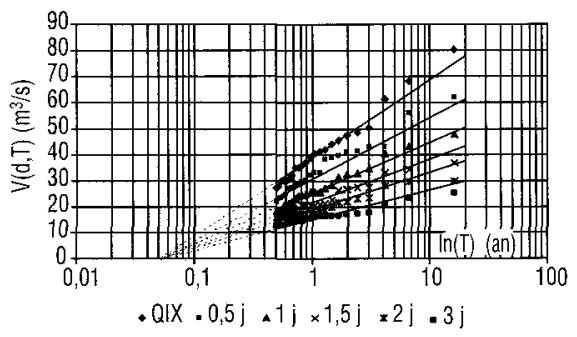

Sous-bassin du Tescou à St-Nauphary $\left(S=287 \mathrm{~km}^{2}\right)$ Modèle QdF sur la chronique E1 : saison hiver 1975-2000

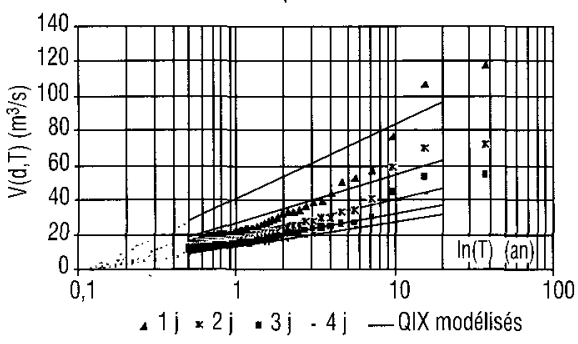

Figure 12 Modélisations QdF- Chroniques Q(t) et E1.

QdF Modeling $-Q(t)$ and $E 1$ time series.

\subsubsection{Tendance des régimes de crue d'hiver du sous-bassin du Tescou}

La tendance sur les régimes de crue peut se déduire d'une simple régression linéaire (figure 13) entre les différents jeux de paramètres précédemment obtenus pour les 4 niveaux d'anthropisation (tableau 4) et référencés d'après le volume théorique prélevé $V$ égal à la médiane des prélèvements cumulés de chaque sous-période (figures $2 \mathrm{~b}$ ). 
Sous-bassin du Tescou : Tendance sur $\Delta$

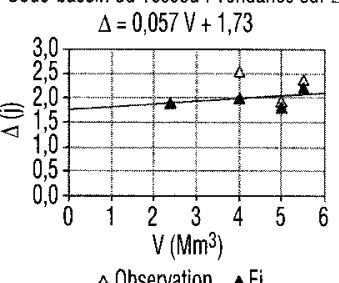

Sous-bassin du Tescou : Tendance sur a $(0)$

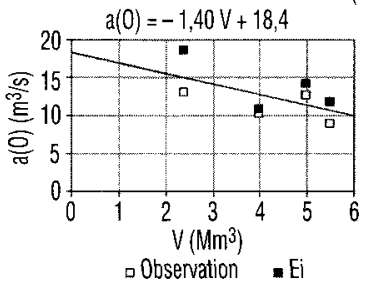

Sous-bassin du Tescou: Tendance sur $\mathrm{X}_{0}(0)$

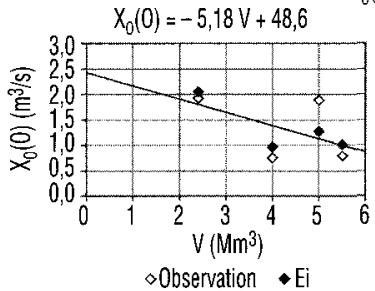

Figure 13 Tendance sur les paramètres des modèles QdF des crues d'hiver du Tescou.

Tendency in QdF model parameters for winter floods in the Tescou sub-basin.

Les trois régressions établies permettent d'estimer chacun des trois paramètres du modèle QdF en fonction du volume théorique stocké dans les retenues collinaires. Le caractère objectif des régimes de crue d'hiver simulés depuis l'état " naturel " $(V=0)$ à l'état d'anthropisation actuel $\left(V=6 \mathrm{Mm}^{3}\right)$ dépend de la qualité des modélisations effectuées, et notamment de la modélisation pluie-débit réalisée à partir de GR4J (chroniques $E_{i}$ ).

Nous insisterons tout particulièrement sur la tendance de $a(0)$ et de $x_{0}(0)$ vers le régime " naturel ", $(V=0)$. Celle-ci est fortement dépendante des modélisations effectuées d'une part pour la chronique de débit observée $Q(t)$ sur la période 1975-1985 (figure 12) et d'autre part pour la chronique $E_{1}$ (1975-2000). L'adéquation de GR4J (calage, validation) sur la sous-période 1975-1985 conditionne tout particulièrement l'extension envisagée 1986-2000 et globalement la représentativité de la chronique stationnaire $E_{1}$ (figure 11) pour le niveau de prélèvement de $2,4 \mathrm{Mm}^{3}$. Au tableau 5 nous montrons pour la souspériode 1975-1985 la cohérence statistique des paramètres de la loi exponentielle calés pour des échantillons sup-seuil tirés des chroniques observée et issue de GR4J (calage + simulation).

Tableau 5 Cohérence statistique des chroniques observée et $E_{1}$ (1975-1985).

Table 5 Statistical coherence of observed and $E_{1}$ time series (1975-1985).

\begin{tabular}{|c|c|c|c|c|c|c|c|}
\hline \multicolumn{8}{|c|}{ Sous-bassin du Tescou : Sous-période stationnaire 1975-1985 } \\
\hline \multicolumn{4}{|c|}{$\begin{array}{l}\text { Chronique de débit } a(t) \text { observée } \\
1975-1985\end{array}$} & \multicolumn{4}{|c|}{$\begin{array}{l}\text { Chronique de débit quotidien associée à GR4J } \\
\text { Calage (1975-1978) + Simulation (1979-1985) }\end{array}$} \\
\hline \multicolumn{4}{|c|}{ Crues d'hiver (mois 12 à 6) } & \multicolumn{4}{|c|}{ Crues d'hiver (mois 12 à 6) } \\
\hline d (j) & seuil $\left(\mathrm{m}^{3} / \mathrm{s}\right)$ & $\mathrm{a}(d) \mathrm{m}^{3} / \mathrm{s}$ & $x_{0}(d)\left(m^{3} / s\right)$ & d (j) & seuil $\left(\mathrm{m}^{3} / \mathrm{s}\right)$ & $a(d) \mathrm{m}^{3} / \mathrm{s}$ & $x_{0}(d)\left(m^{3} / s\right)$ \\
\hline 1 & 17,9 & 8,97 & 25,1 & 1 & 16,1 & 8,79 & 22,9 \\
\hline 2 & 13,6 & 5,60 & 17,9 & 2 & 14,4 & 4,52 & 17,9 \\
\hline 3 & 12,5 & 3,35 & 15,2 & 3 & 11,3 & 3,86 & 14,5 \\
\hline
\end{tabular}




\subsubsection{Impact des retenues collinaires sur les quantiles de crue d'hiver du Tescou et de la Séoune}

Pour le sous-bassin du Tescou et à partir des régressions linéaires précédentes nous pouvons associer à chaque niveau de prélèvement théorique un modèle QdF à 3 paramètres. Une représentation quantitative de l'impact sur les quantiles de crue de pointe et de $24 \mathrm{~h}$ par exemple peut être ensuite proposée (figure 14).

Une démarche analogue menée sur les chroniques de crue d'hiver du sousbassin de la Séoune permet de proposer la représentation suivante (figure 15).

Par ailleurs, la tendance présentée précédemment sur les quantiles d'hiver "naturels " de un jour et quatre jours (figures 14 et 15) reste cohérente en regard du volume théorique actuellement prélevé, proche de $6 \mathrm{Mm}^{3}$ (figure 16). Cela est de même vrai pour deux jours et huit jours, durées compatibles avec la dynamique de crue des sous-bassins.

Sous-bassin du Tescou

Impact sur les débits de pointe des crues d'hiver

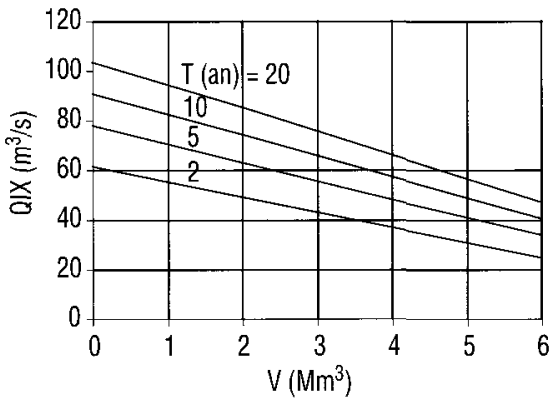

Sous-bassin du Tescou

Impact sur les débits de $24 \mathrm{~h}$ des crues d'hiver

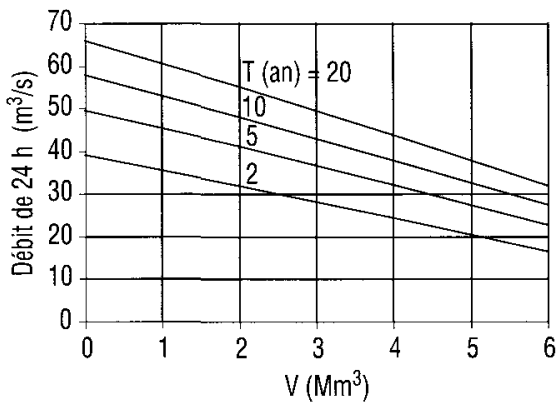

Figure 14 Impact sur les quantiles d'hiver de pointe et de $24 \mathrm{~h}$ du sous-bassin du Tescou.

Impact on winter peak flood and 24-h quantiles of the Tescou sub-basin.

Sous-bassin de la Séoune

Impact sur les débits de pointe des crues d'hiver

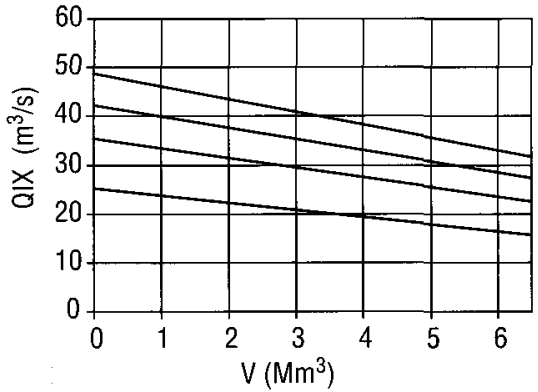

Sous-bassin de la Séoune

Impact sur les débits de 4 jours des crues d'hiver

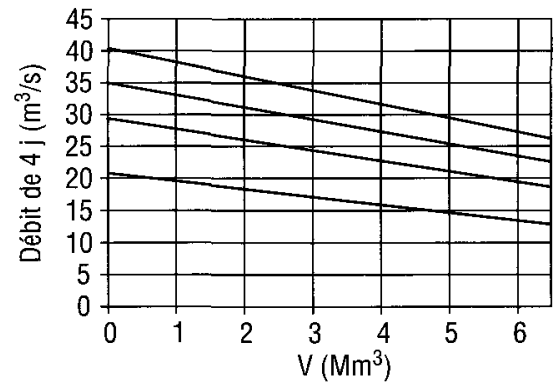

Figure 15 Impact sur les quantiles d'hiver de pointe et de 4 jours du sous-bassin de la Séoune.

Impact on winter peak flood and 4-days quantiles for the Séoune subbasin. 
Sous-bassin du Tescou Cohérence de l'impact sur les quantiles $V(d, T)$

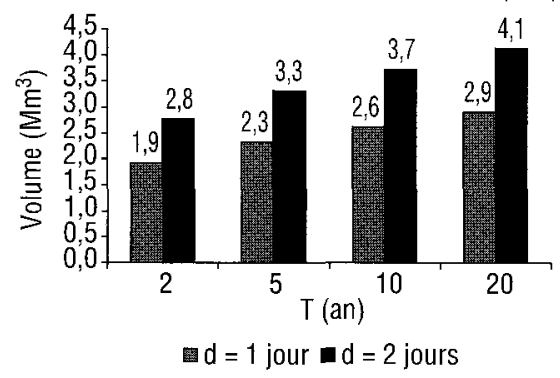

Sous-bassin de la Séoune Cohérence de l'impact sur les quantiles $V(d, T)$

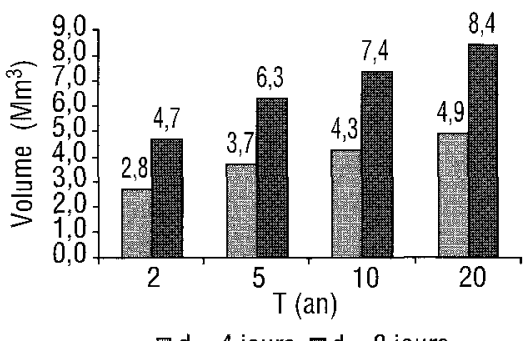

Figure 16 Cohérence de l'impact sur les quantiles d'hiver « naturels ».

Coherence of the impact on the "natural" winter quantiles.

\subsubsection{Conclusion partielle sur le régime des crues d'hiver du Tescou et de la Séoune}

Les modélisations effectuées ont permis de montrer l'évolution des régimes de crue d'hiver des deux sous-bassins en fonction de la pression des prélèvements. Les crues de la saison hiver du sous-bassin du Tescou sont fortement réduites en pointe et volume par l'ensemble des petites retenues, de même que celles du sous-bassin de la Séoune dans une moindre mesure. Le temps de transfert $\Delta$ pour ce qui concerne le Tescou, bassin rapide aux crues peu volumineuses, est sensiblement augmenté d'environ $8 \mathrm{~h}$ entre l'état de prélèvement nul (« naturel » : $\Delta=1,73 \mathrm{j})$ et l'état de prélèvement actuel proche de $6 \mathrm{Mm}^{3}(\Delta=$ 2,07 j). Pour le sous-bassin de la Séoune par contre, les crues de grande inertie et volumineuses font que $\Delta$ reste pratiquement inchangé $(\Delta=19$ j environ) entre l'état « naturel » et l'état de prélèvement actuel proche de $6,5 \mathrm{Mm}^{3}$.

\section{4 - IMPACT DES PRÉLÈVEMENTS POUR L'IRRIGATION SUR LES MODULES SAISONNIERS DES SOUS-BASSINS DE LA SÉOUNE ET DU TESCOU}

Comme pour les crues, nous ferons la distinction entre module annuel d'hiver $Q A \_H$ (mois 12 à 6) et module annuel d'été $Q A \_E$ (mois 7 à 11). Aux modules saisonniers nous associerons les coefficients d'écoulement, respectivement $C E \_H$ et $C E \_E$. Nous rappelons que le module saisonnier correspond à la moyenne des débits journaliers sur la saison considérée.

\subsection{Identification de ruptures de stationnarité des chroniques QA_E et QA_H}

Pour détecter les ruptures de stationnarité des chroniques $Q A \_E$ et $Q A \_H$ observées nous utilisons une adaptation du test de l'ellipse de Bols (1986). 


\subsubsection{Rappel sur le test de Bols et adaptation}

À l'origine, l'ellipse de Bols sert à vérifier l'homogénéité d'une série chronologique $\left\{Y_{i}\right\}_{i=1 . n}$ à l'aide des données $\left\{X_{i}\right\}_{i=1 . n}$ d'une série de référence homogène. Une courbe de régression linéaire est ajustée entre les séries $X$ et $Y$, et telle que $Y_{i}=a \cdot X_{i}+b+\varepsilon_{i}, \forall \mathrm{i}, \mathrm{i}=1$... $\mathrm{N}$.

Les $\left\{\varepsilon_{i}\right\}_{i=1 . . n}$ constituent la série des résidus de la régression linéaire, de moyenne nulle et d'écart type $s_{e}=S_{y} \sqrt{1-r_{x y}{ }^{2}}$ où $S_{y}$ est l'écart type de la série $Y$ et $r_{x y}$ est le coefficient de corrélation entre $X$ et $Y$.

Soit $S E_{k}=\sum_{i=1}^{k} \varepsilon_{i}$ la série des cumuls des résidus de la régression linéaire, d'écart type (9).

$$
\sigma\left[S E_{k}\right]=s_{e} \sqrt{\frac{k \cdot(N-k)}{N-1}}
$$

L'intervalle de confiance de cette série est une ellipse dont l'axe principal coïncide avec celui des abscisses et dont les bornes pour chaque valeur de $k$ sont définies selon (10).

$$
[I . C(\%)]_{k}= \pm \alpha(f) \cdot \sigma\left[S E_{k}\right]
$$

où :

$\alpha(\phi)$ est la variable centrée réduite de Gauss (1,64 pour un I.C. à $90 \%)$

Pour appliquer ce test à la seule chronique de la variable échantillonnée nous substituerons par analogie la série des cumuls des résidus de la régression par la série des cumuls des écarts des valeurs échantillonnées à leur moyenne, d'écart type (11). L'application nécessite par ailleurs que l'échantillon soit gaussien ou rendu comme tel.

$$
\sigma\left(S E_{k}\right)=\sigma_{\text {échantilon }} \sqrt{\frac{k \cdot(N-k)}{N-1}}
$$

où :

$\sigma_{\text {échantillon }}$ est l'écart type des valeurs échantillonnées.

\subsubsection{Modules d'été QA_E des sous-bassins de la Séoune et du Tescou}

Les modules d'été $Q A \_E$ et les coefficients d'écoulement correspondant à la pluie annuelle d'été ( $C E \_E=Q A \_E / P A_{-} E$ ) sont stationnaires sur la période observée (1969-2003). Les cumuls des écarts des valeurs échantillonnées par rapport à leur moyenne sont compris en général dans l'ellipse à $90 \%$ de BOIs (figure 17), on constate un seul point sortant (cf. 3.1.2). II n'y a donc pas d'impact des éventuels pompages ou compléments de remplissage des retenues collinaires sur les modules d'été. 

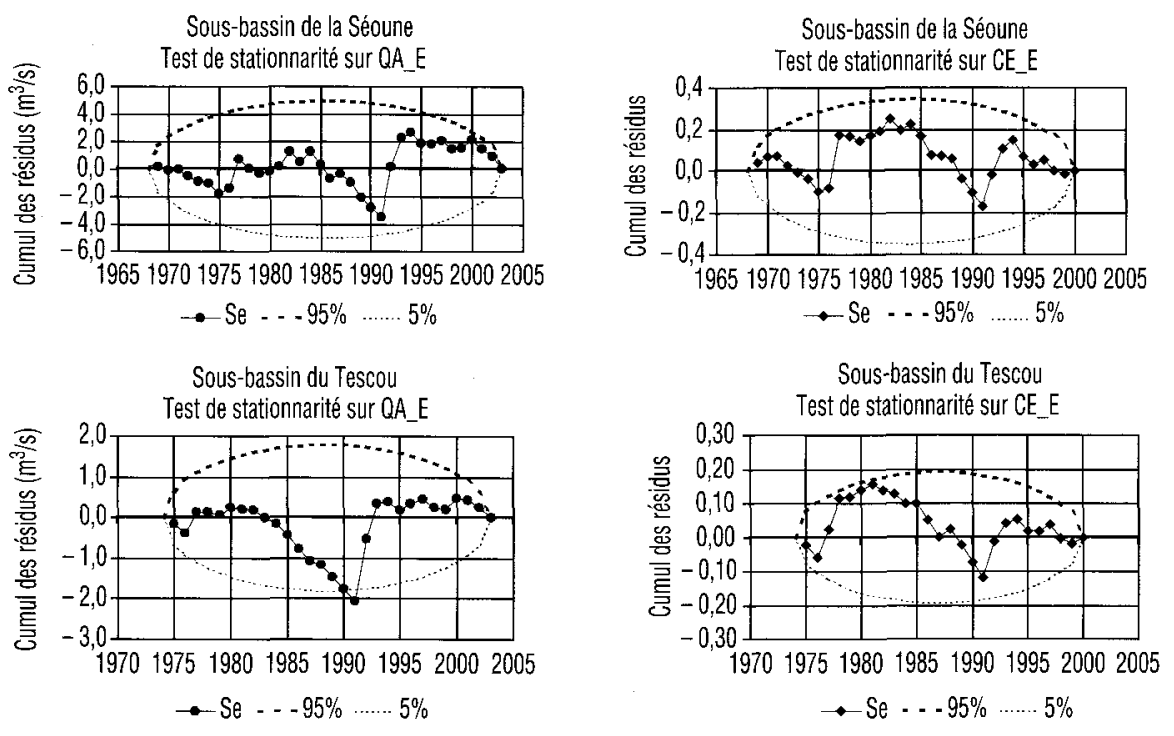

Figure 17 Stationnarité des modules d'été des sous-bassins.

Stationnarity of mean summer discharge time series for the subbasins.

\subsubsection{Modules d'hiver QA_H des sous-bassins de la Séoune et du Tescou}

Pour les deux sous-bassins le test de Bols permet d'identifier une rupture de stationnarité pour la chronique $Q A \_H$ (figure 18). Pour le sous-bassin du Tescou comme celui de la Séoune, par exemple, la pluie moyenne $P A \_H$ apparaît stationnaire, ce qui conforte l'impact anthropique sur le coefficient d'écoulement (figure 19).

Par approches successives, le test de Bols nous permet de définir pour les deux sous-bassins deux sous-périodes stationnaires des modules d'hiver (figure 20).
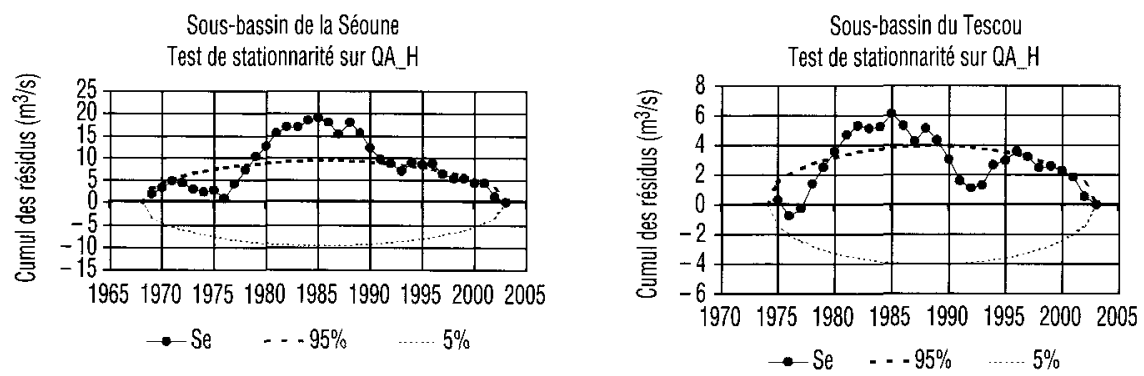

Figure 18 Rupture de stationnarité des modules d'hiver des sous-bassins de la Séoune et du Tescou.

Stationnarity break in mean annual winter discharge time series for the Séoune and Tescou sub-basins. 
Sous-bassin de la Séoune

Test de stationnarité sur PA_H

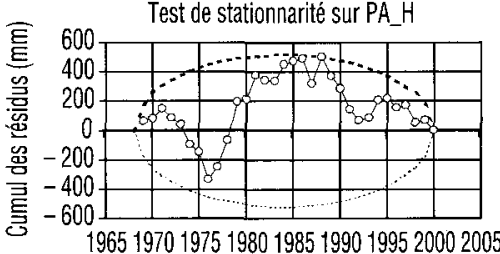

$\multimap$ Se $\quad \ldots-95 \% \quad 5 \%$
Sous-bassin de la Séoune

Test de stationnarité sur CE_H

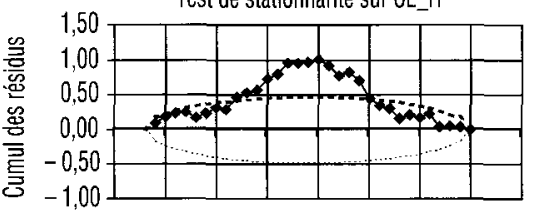

196519701975198019851990199520002005

$\rightarrow$ Se $\quad-.95 \% \quad \ldots . .5 \%$

Figure 19 Consolidation de l'impact anthropique sur le coefficient d'écoulement d'hiver.

Consolidation of anthropogenic impacts on the winter runoff coefficient.

Sous-bassin de la Séoune

Stationnarité des QA H sur 1969-1985

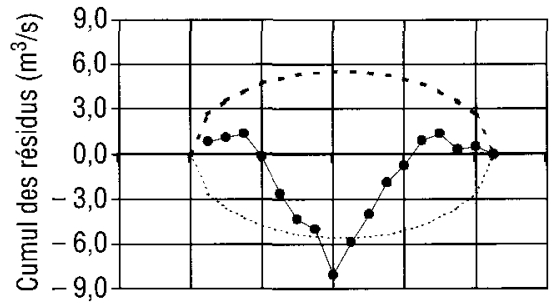

1964196819721976198019841988

$\rightarrow \mathrm{Se} \quad-.95 \% \quad \ldots .5 \%$

Sous-bassin du Tescou Stationnarité des QA_H sur 1975-1985

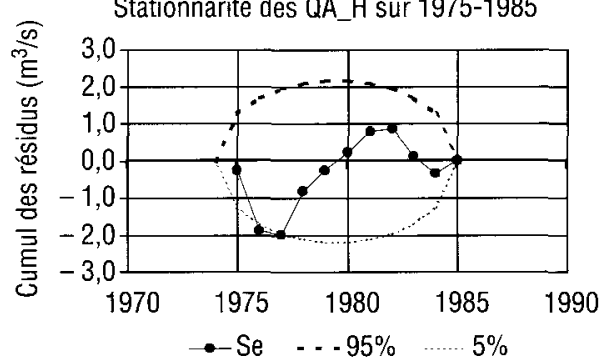

Sous-bassin de la Séoune

Stationnarité des QA-H sur 1986-2003

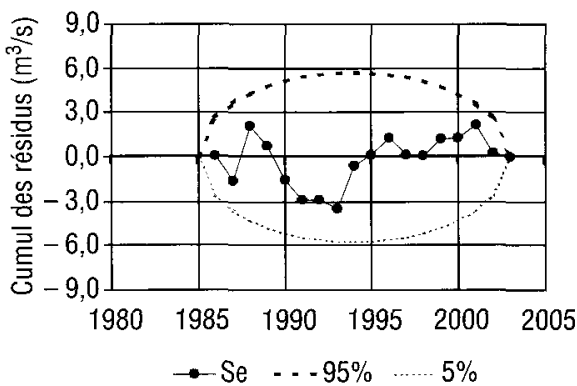

Sous-bassin du Tescou

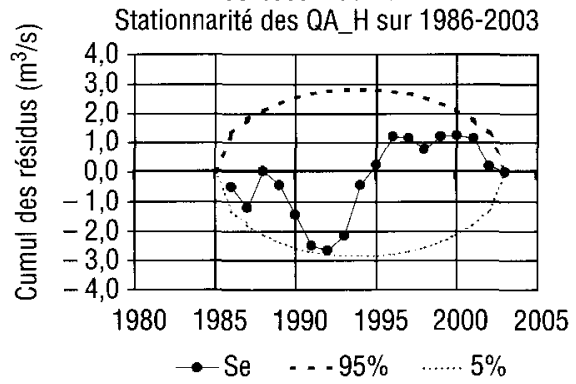

Figure 20 Identification des sous-périodes stationnaires pour les deux sousbassins.

Identification of stationary sub-periods for both sub-basins. 


\subsection{Analyse fréquentielle des modules saisonniers des sous-bassins de la Séoune et du Tescou}

\subsubsection{Modules d'été}

Pour les modules d'été, aucune rupture de stationnarité n'a été identifiée. Sur chacun des échantillons $Q A \_E$ relatifs aux chroniques observées nous avons choisi d'ajuster une loi de Weibull à deux paramètres (12) pour rendre compte des faibles valeurs des modules d'été (tableau 6 et figure 21).

$$
Q A_{-} E\left(U_{F}\right)=\beta U_{F}^{t / \alpha}
$$

avec :

$\mathrm{U}_{\mathrm{F}}=-\ln (1-\mathrm{F})$

$\beta$ : paramètre d'échelle, $\left(\mathrm{m}^{3} / \mathrm{s}\right)$.

$\alpha:$ paramètre de forme.

$F_{i}=\frac{i-0,3}{n+0,4}$, probabilité au non-dépassement, $i$ : rang et $n:$ nombre d'années d'observation.

À partir de l'équation (12) et des paramètres établis (tableau 6), nous pouvons estimer le quantile de l'année moyenne $(T=2)$, les quantiles dits « secs " pour $F<0,5(T=1 / F)$ et les quantiles dits « humides » pour $F>0,5(T=1 /(1-F))$. Nous en donnons une représentation avec leur intervalle de confiance à $90 \%$ (figure 21).

Tableau 6 Quantiles des modules d'été des sous-bassins.

Table 6 Mean summer discharge quantiles for the sub-basins.

\begin{tabular}{|c|c|c|c|c|c|}
\hline \multicolumn{6}{|c|}{$\begin{array}{l}\text { Sous-bassin de la Séoune : Quantiles QA E(T) } \\
\text { Paramètres de la loi de Weibull : } \alpha=1,224 \beta=\overline{1}, 227\left(\mathrm{~m}^{3} / \mathrm{s}\right)\end{array}$} \\
\hline $\mathbf{T}(\mathrm{an})$ & \multicolumn{2}{|c|}{ année sèche } & année moyenne & année humide & $\begin{array}{r}\text { mide } \\
10\end{array}$ \\
\hline$U_{F}$ & 0,105 & 0,223 & 0,693 & 1,61 & 2,30 \\
\hline $95 \%$ & 0,300 & 0,510 & 1,15 & 2,22 & 3,00 \\
\hline Valeur centrée & 0,195 & 0,360 & 0,909 & 1,81 & 2,42 \\
\hline $5 \%$ & 0,090 & 0,210 & 0,670 & 1,40 & 1,85 \\
\hline \multicolumn{6}{|c|}{$\begin{array}{l}\text { Sous-bassin du Tescou : Quantiles QA E(T) } \\
\text { Paramètres de la loi de Weibull : } \alpha=1,251 \beta=0,45\left(\mathrm{~m}^{3} / \mathrm{s}\right)\end{array}$} \\
\hline$T(\operatorname{an})$ & 10 & $\begin{array}{r}5 \text { che } \\
5\end{array}$ & $\begin{array}{l}\text { année moyenne } \\
2\end{array}$ & $\begin{array}{c}\text { anné } \\
5\end{array}$ & $\begin{array}{r}\text { mide } \\
10\end{array}$ \\
\hline$U_{F}$ & 0,105 & 0,223 & 0,693 & 1,61 & 2,30 \\
\hline $95 \%$ & 0,110 & 0,190 & 0,430 & 0,820 & 1,10 \\
\hline Valeur centrée & 0,070 & 0,140 & 0,340 & 0,660 & 0,880 \\
\hline $5 \%$ & 0,030 & 0,080 & 0,240 & 0,500 & 0,660 \\
\hline
\end{tabular}


Sous-bassin de la Séoune

Loi de Weibull ajustée aux modules d'été observés

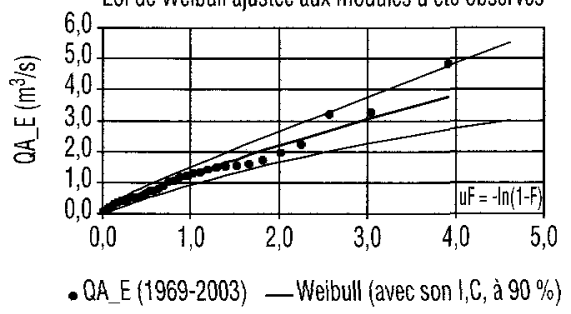

Sous-bassin du Tescou

Loi de Weibull ajustée aux modules d'été observés

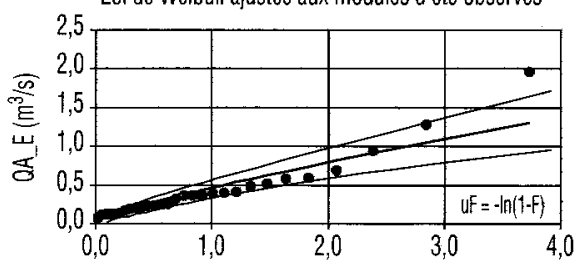

- QA_E (1975-2003) - Weibull (avec son 1,C, à $90 \%$ )

Figure 21 Loi de Weibull ajustée sur les QA_E observés des sous-bassins.

Weibull distribution adjusted for the observed $Q A \_E$ samples of the sub-basins.

Nota :

Le soutien d'étiage prévu de l'ordre de $2 \mathrm{Mm}^{3}$ pour le sous-bassin du Tescou (CACG, 2001) correspondrait à un débit injecté continûment de $0,151 \mathrm{~m}^{3} / \mathrm{s}$

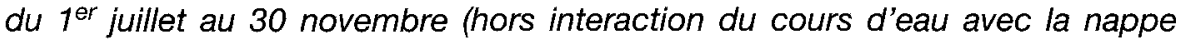
d'accompagnement). Son efficacité est significative en regard des quantiles «secs " du tableau 6.

L'absence d'impact sur les modules d'été des sous-bassins de la Séoune et du Tescou est cohérente avec les résultats trouvés pour les crues d'été qui expliquent généralement pour une grande part la ressource des modules.

\subsubsection{Modules d'hiver}

\subsubsection{Constitution des chroniques homogènes étendues $M 1$ et $M 2$}

Pour les sous-bassins du Tescou et de la Séoune deux sous-périodes stationnaires ont été identifiées (figure 20). L'extension des chroniques de débit journalier de ces sous-périodes à la durée d'observation de la pluie est obtenue à partir du modèle pluie-débit GR4J. L'efficience du calage du modèle pour chacune des sous-périodes stationnaires conditionne cette extension (tableau 7).

Tableau 7 Efficience du modèle GR4J (saison année).

Table 7 Efficiency of the GR4J model (year season).

\begin{tabular}{cccc}
\hline \multicolumn{2}{c}{ Sous-bassin du Tescou } & \multicolumn{2}{c}{ Sous-bassin de la Séoune } \\
\hline Sous-période 1975-1985 & Sous-période 1986-2000 & Sous-période 1969-1985 & Sous-période 1986-2000 \\
Calage GR4J : 1975-1978 & Calage GR4J 1992-1993 & Calage GR4J : 1979-1981 & Calage GR4J : 1994-1996 \\
Nash (sur Q) : 78 \% & Nash (sur Q) : 84 \% & Nash (sur Q) : $90 \%$ & Nash (sur Q) : 92 \% \\
\hline
\end{tabular}

Pour le sous-bassin du Tescou, par exemple, les jeux de paramètres associés aux sous-périodes 1975-1985 et 1986-2000 sont respectivement ceux déjà retenus pour les chroniques $E_{1}$ et $E_{3}$ (tableau 4). La figure 22 permet de valider l'adéquation du modèle GR4J. 
Sous-bassin du Tescou

sous-période stationnaire 1975-1985

Calage et validation du Modèle GR4J

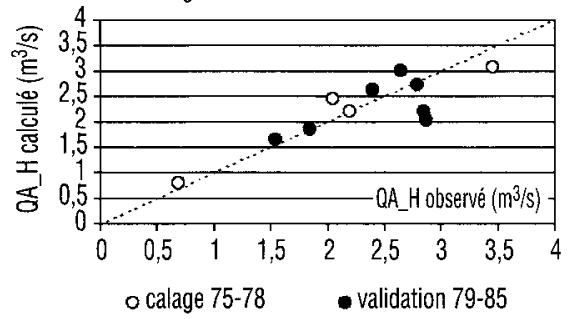

Sous-bassin du Tescou

sous-période stationnaire 1986-2000

calage et validation du modèle GR4J

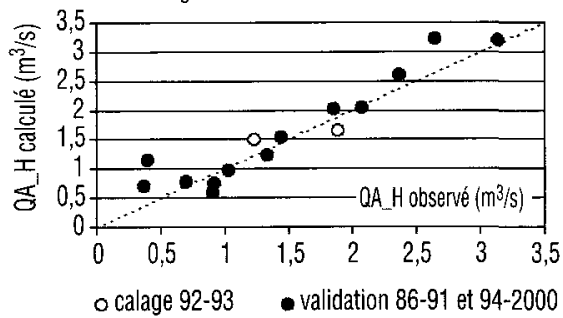

Figure 22 Calage et validation de GR4J sur les sous-périodes stationnaires des QA_H du Tescou.

Calibration and validation of the GR4J model on QA_H stationary subperiods for the Tescou sub-basin.

Par ailleurs, pour conforter l'homogénéité des chroniques étendues par simulation, nous avons vérifié l'équivalence des coefficients d'écoulements moyens $\overline{C E} E_{-} H$ entre sous-périodes stationnaires et extensions obtenues par simulation. Soit $M_{1}$ (équivalente à $E 1$ ) et $M_{2}$ les chroniques étendues caractérisant respectivement l'état « naturel » et l'état d'anthropisation actuel des sousbassins (tableau 8 ).

Tableau 8 Homogénéité des chroniques $M_{1}$ et $M_{2}$.

Table 8 Homogeneity of the $M_{1}$ and $M_{2}$ time series.

\begin{tabular}{|c|c|c|c|c|c|c|c|c|c|c|c|c|c|c|}
\hline \multicolumn{7}{|c|}{ Sous-bassin du Tescou } & \multicolumn{8}{|c|}{ Sous-bassin de la Séoune } \\
\hline \multicolumn{3}{|c|}{$M_{1}(1975-2000)$} & \multicolumn{4}{|c|}{$\mathrm{M}_{2}(1975-2000)$} & \multicolumn{4}{|c|}{$M_{1}(1969-2000)$} & \multicolumn{4}{|c|}{$\mathrm{M}_{2}(1969-2000)$} \\
\hline $\begin{array}{l}\text { Observation } \\
1975-1985\end{array}$ & \multicolumn{2}{|c|}{$\begin{array}{l}\text { Simulation } \\
\text { 1986-2000 }\end{array}$} & \multicolumn{2}{|c|}{$\begin{array}{c}\text { Observation } \\
1986-2000\end{array}$} & \multicolumn{2}{|c|}{$\begin{array}{l}\text { Simulation } \\
\text { 1975-1985 }\end{array}$} & \multicolumn{2}{|c|}{$\begin{array}{c}\text { Observation } \\
1969-1985\end{array}$} & \multicolumn{2}{|c|}{$\begin{array}{l}\text { Simulation } \\
1986-2000\end{array}$} & \multicolumn{2}{|c|}{$\begin{array}{c}\text { Observation } \\
1986-2000\end{array}$} & \multicolumn{2}{|c|}{$\begin{array}{l}\text { Simulation } \\
1969-1985\end{array}$} \\
\hline$\overline{\overline{\mathrm{CE}} \mathrm{H}} \mathrm{S}$ & $\overline{\overline{C E} B}$ & $s$ & $\overline{\overline{C E} \_H}$ & $s$ & $\overline{\overline{\mathrm{CE}} \_\mathrm{H}}$ & $s$ & $\overline{\mathrm{CE} B \mathrm{H}}$ & $s$ & $\overline{\mathrm{CE} \_\mathrm{H}}$ & $s$ & $\overline{\mathrm{CE} H}$ & $s$ & $\overline{\overline{C E} \_H}$ & $s$ \\
\hline $0,32 \quad 0,058$ & 0,33 & 0,088 & 0,19 & 0,076 & 0,19 & 0,049 & 0,43 & 0,065 & 0,40 & 0,094 & 0,30 & 0,091 & 0,28 & 0,054 \\
\hline
\end{tabular}

4.2.2.2 Incohérence des coefficients d'écoulements et des volumes théoriques stockés

Pour le sous-bassin de la Séoune et pour une même pluie observée $P A \_H$ stationnaire (1969-2000), le coefficient d'écoulement moyen d'hiver passe de 0,42 pour l'état « naturel » à 0,29 pour l'état anthropisé (prélèvement théorique de $6,5 \mathrm{Mm}^{3}$ ), soit une diminution de $31 \%$ en moyenne. Par référence à la pluie annuelle moyenne d'hiver de $503 \mathrm{~mm}$ cela équivaut à un déficit d'environ $30 \mathrm{Mm}^{3}$. De toute évidence, en regard des $6,5 \mathrm{Mm}^{3}$ théoriques stockés, d'autres facteurs influencent majoritairement les modules d'hiver. Cela expliquerait la rupture de stationnarité observée et qui selon toute vraisemblance ne serait pas apparue sur la chronique des modules d'hiver pour les seuls besoins de l'irrigation.

Le même constat peut être fait pour le sous-bassin du Tescou. Le coefficient d'écoulement moyen d'hiver passe de 0,33 pour l'état « naturel » à 0,19 pour l'état anthropisé (prélèvement théorique de $5,7 \mathrm{Mm}^{3}$ ), soit une diminution de $42 \%$ en moyenne. Par référence à la pluie annuelle moyenne d'hiver (19752000) de $465 \mathrm{~mm}$ cela équivaut à un déficit d'environ $18,5 \mathrm{Mm}^{3}$. C'est plus de trois fois le volume théorique stocké pour l'irrigation $\left(5,7 \mathrm{Mm}^{3}\right)$. 
II n'y a aucune commune mesure entre ces déficits et les volumes théoriques stockés à partir des retenues collinaires. Ils sont pourtant consécutifs à l'anthropisation des régimes naturels des sous-bassins dont les volumes théoriques des retenues collinaires ne constituent qu'un des facteurs. Les résultats statistiques présentés ci-après traduisent donc l'impact global de l'anthropisation des régimes hydrologiques.

\subsubsection{Modélisation statistique}

Sur chacun des échantillons $Q A \_H$ relatifs aux chroniques $M_{1}$ et $M_{2}$ nous avons ajusté une loi de Weibull à deux paramètres pour rendre compte des faibles valeurs des modules d'hiver (tableau 9). Les modules d'hiver estimés à partir de la chronique $M_{2}$ devraient être pris en considération pour des objectifs de gestion actuels ou à venir de la ressource, si les conditions climatiques et d'occupation du sol n'évoluent guère.

Tableau 9 Paramètres de la loi de Weibull ajustés sur les modules d'hiver des sous-bassins.

Table 9 Parameters of the Weibull distribution adjusted on the mean winter discharge from the sub-basins.

\begin{tabular}{ccccccccc}
\hline \multicolumn{8}{c}{ Ajustement d'une loi de Weibull à 2 paramètres } \\
\hline \multicolumn{3}{c}{ Sous-bassin du Tescou } & \multicolumn{4}{c}{ Sous-bassin de la Séoune } \\
\hline \multicolumn{2}{c}{ Chronique $\mathbf{M}_{\mathbf{1}}$} & \multicolumn{2}{c}{ Chronique $\mathbf{M}_{\mathbf{2}}$} & \multicolumn{2}{c}{ Chronique $\mathbf{M}_{\mathbf{1}}$} & \multicolumn{2}{c}{ Chronique $\mathbf{M}_{\mathbf{2}}$} \\
\hline $\boldsymbol{\alpha}$ & $\boldsymbol{\beta}$ & $\boldsymbol{\alpha}$ & $\boldsymbol{\beta}$ & $\boldsymbol{\alpha}$ & $\boldsymbol{\beta}$ & $\boldsymbol{\alpha}$ & $\boldsymbol{\beta}$ \\
\hline 2,633 & 2,744 & 2,245 & 1,611 & 3,517 & 5,934 & 3,061 & 4,185 \\
\hline
\end{tabular}

Nous donnons à la figure 23 une représentation des quantiles d'hiver $Q A \_H(T)$ et de leur intervalle de confiance à $90 \%$ déduits de l'ajustement de la loi de Weibull (tableau 9).

\subsection{Conclusion partielle}

À partir des résultats statistiques précédents (tableau 9 et figure 23) on retrouve bien pour les quantiles d'hiver, de l'année moyenne en particulier, les déficits de $30 \mathrm{Mm}^{3}$ (Séoune) et de $18,5 \mathrm{Mm}^{3}$ (Tescou) précédemment estimés à partir des coefficients d'écoulement. Le fait de ne pas avoir constaté d'impact sur les crues et les modules d'été pour les deux sous-bassins nous permet de dire que la ressource nécessaire à l'irrigation durant la saison été (mois 7 à 11) dépend essentiellement du volume d'eau stocké par les retenues collinaires pendant la saison hiver (mois 12 à 6). Pour ce qui concerne les modules d'hiver, le fort impact sur les coefficients d'écoulements est consécutif à l'anthropisation des régimes d'écoulement mais ne peut pas être expliqué par les seules retenues collinaires. En effet, et selon toute vraisemblance, l'usage de l'eau pour l'irrigation à hauteur des volumes théoriques stockés ne devrait pas induire de rupture de stationnarité significative sur les chroniques de module d'hiver des sous-bassins, contrairement à ce qui est observé (figure 18). 


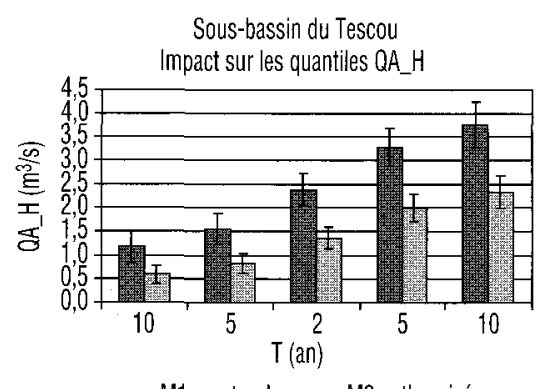

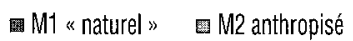

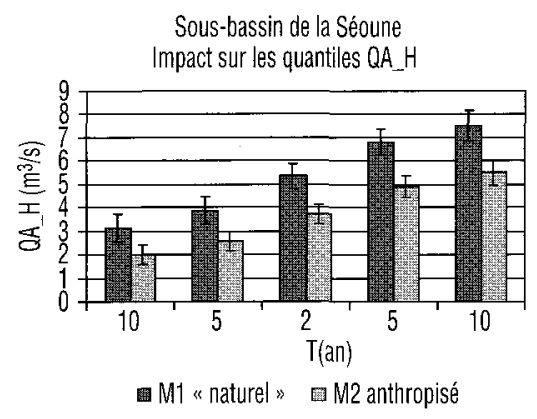

Sous-bassin de la Séoune Impact sur les quantiles QA_H

Figure 23 Quantiles et I.C. à $90 \%$ des modules d'hiver des sous-bassins " naturels » et anthropisés.

Quantiles and $90 \%$ confidence intervals for mean annual winter discharge from "natural" and anthropogenic sub-basins.

\section{5 - IMPACT DES PRÉLÈVEMENTS SUR LES ÉTIAGES DES SOUS-BASSINS DE LA SÉOUNE ET DU TESCOU}

Nous rappelons que le QMNA5 « débit mensuel minimum annuel d'étiage de période de retour quinquennale sèche " est la norme de débit à laquelle se réfèrent les gestionnaires (loi sur l'eau de janvier 1992). Nous avons préféré considérer une origine variable de la période de 30 jours plutôt qu'une origine calendaire fixe. II s'agit des $V C N_{30}$ " débit moyen minimum annuel de 30 jours consécutifs ". L'origine variable de la période de 30 jours montre une différenciation significative des échantillons QMNA « débit mensuel minimum annuel d'étiage " et VCN30 constitués (figure 24).

L'échantillonnage selon le découpage calendaire des QMNA a tendance à surestimer les débits moyens minimum sur 30 jours consécutifs définis à partir des $V C N_{30}$. Autrement dit, la moyenne mobile est plus pertinente que le découpage calendaire.

Sous-bassin de la Séoune Relation entre VCN $_{30}$ et QMNA (1969-2003)

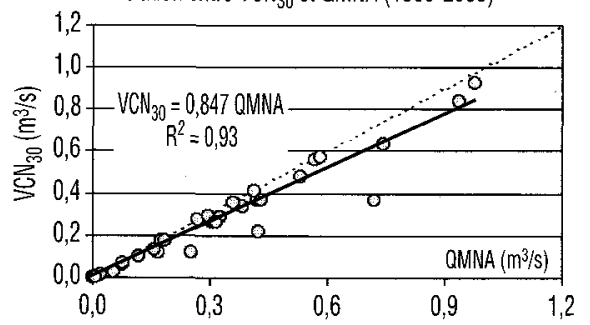

Sous-bassin du Tescou Relation entre $\mathrm{VCN}_{30}$ et QMNA (1975-2003)

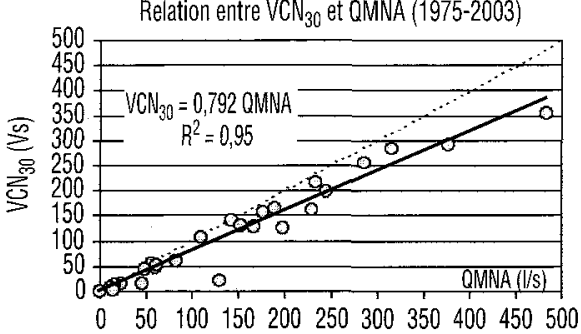

Figure 24 Comparaison des échantillons $V_{C N}$ et $Q M N A$. Comparison of $\mathrm{VCN}_{30}$ and $\mathrm{QMNA}$ samples. 


\subsection{Identification d'une éventuelle rupture de stationnarité sur les chroniques de $\mathrm{VCN}_{30}$}

Seule la probabilité liée au rang de chaque valeur de $V C N_{30}$ (valeur nulle comprise) est considérée. Cette probabilité associée à une loi normale de moyenne nulle et d'écart-type 1 permet ensuite d'en déduire l'ellipse de BoIs avec son intervalle de confiance à $90 \%$ (figure 25). Aucune rupture de stationnarité n'est identifiable.

Sous-bassin de la Séoune

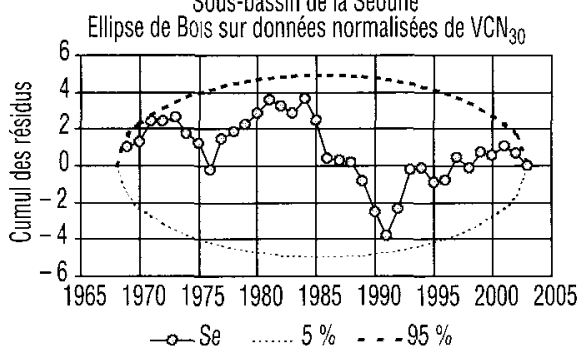

Sous-bassin du Tescou

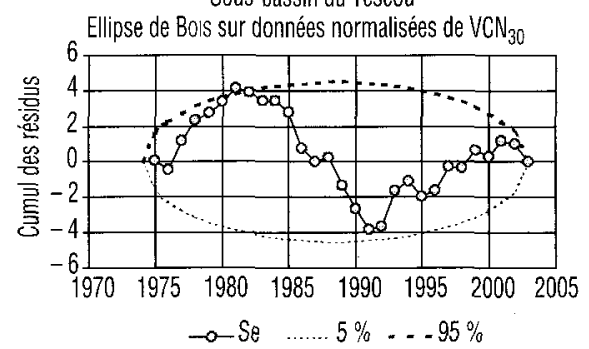

Figure 25 Ellipse de BoIs sur les échantillons normalisés de $\mathrm{VCN}_{30}$.

Bols ellipse in $\mathrm{VCN}_{30}$ standardized samples.

\subsection{Ajustements statistiques sur les chroniques $\mathrm{VCN}_{30}$ observées}

À toutes fins utiles, nous avons ajusté une loi de Weibull sur les échantillons de $\mathrm{VCN}_{30}$ tronqués des valeurs nulles (figure 26 ).

Après correction des fréquences, nous présentons au tableau 10 les principaux quantiles dont le $\mathrm{VCN}_{30}(5)$ et leur intervalle de confiance à $90 \%$.

Sous-bassin de la Séoune Loi de Weibull ajustée aux étiages de 30 jours

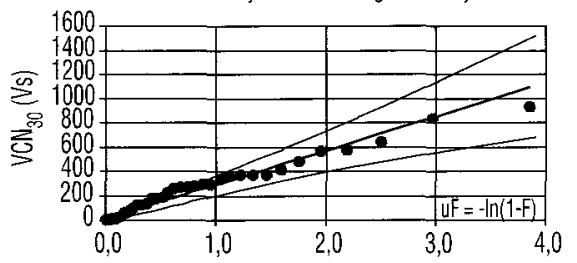

- VCN30 (1969-2003) —Weibull (avec son I,C, à $90 \%)$
Sous-bassin du Tescou Loi de Weibull ajustée aux étiages de 30 jours

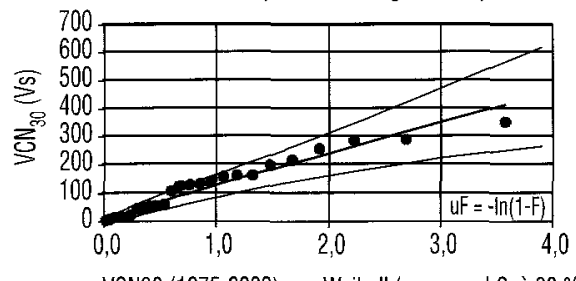

- VCN30(1975-2003) —Weibull (avec son I,C, à $90 \%)$

Figure 26 Loi de Weibull ajustée sur les échantillons tronqués des VCN30 observés des sous-bassins.

Weibull distribution adjusted for truncated VCN30 samples in the subbasins. 
Tableau 10 Quantiles des $\mathrm{VCN}_{30}$ (T) et I.C. à $90 \%$ des sous-bassins.

Table 10 Quantiles and $90 \%$ confidence intervals for $V C N_{30}(T)$ of the subbasins.

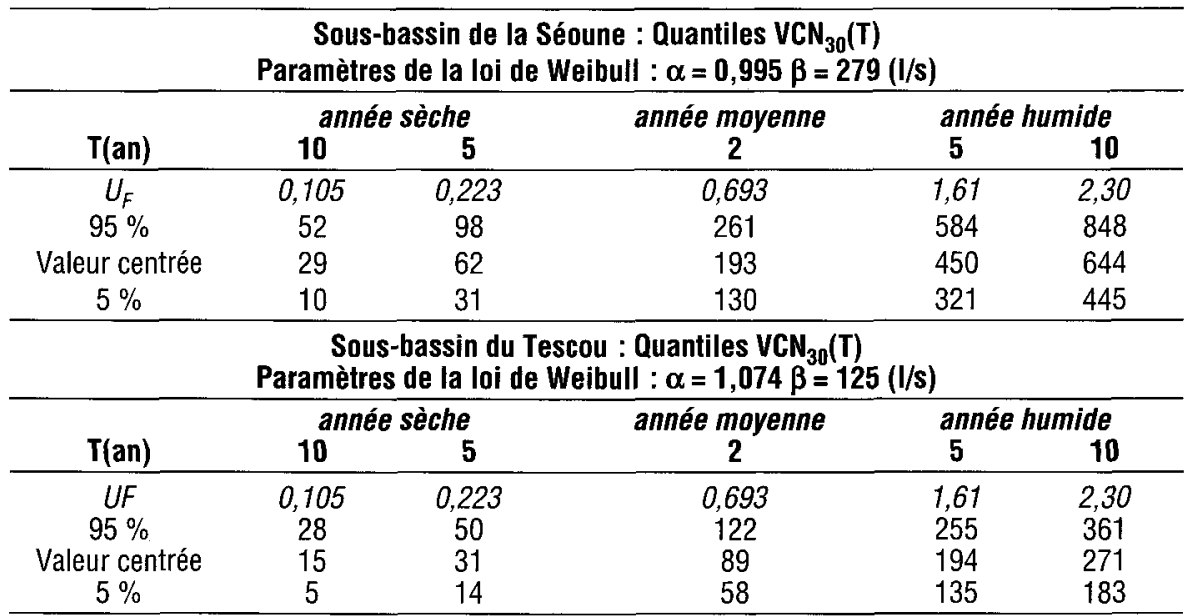

\subsection{Conclusion partielle}

II ne semble pas y avoir d'impact des éventuels pompages sur les débits d'étiage des sous-bassins du Tescou et de la Séoune. De manière générale, la variabilité climatique est déterminante pour expliquer la plus ou moins grande sévérité des étiages.

Le soutien d'étiage de $2 \mathrm{Mm}^{3}\left(151 \mathrm{l} / \mathrm{s}\right.$ injecté en continu du $1^{\text {er }}$ juillet au 30 novembre) prévu pour le Tescou aurait pour conséquence un glissement en fréquence du $V C N_{30}(5)$ du quinquennal « sec » vers le quinquennal « humide », si l'on s'en réfère aux estimations données au tableau 10.

\section{6 - CONCLUSION GÉNÉRALE}

Nous avons étudié l'impact de l'usage de l'eau pour l'irrigation sur trois composantes du régime hydrologique des sous-bassins de la Séoune et du Tescou: crues, modules et étiages. Une analyse saisonnalisée hiver (mois 12 à 6) et été (mois 7 à 11) des chroniques de débit observées et les tests de ruptures de stationnarité correspondants nous ont permis de différencier les impacts sur le régime hydrologique des sous-bassins. Cette analyse menée sur les débits a été effectuée systématiquement sur les pluies pour consolider nos résultats.

De manière générale, les crues de la saison hiver des deux sous-bassins sont fortement réduites en pointe et volume par l'ensemble des petites retenues. Ces dernières sont sans action significative sur le temps de transfert 
( $\Delta=19 j$ environ) du sous-bassin de la Séoune. Ce résultat est logique du fait de ses crues volumineuses et de grande inertie. Pour le Tescou par contre, aux crues rapides et peu volumineuses, $\Delta$ augmente sensiblement de $8 \mathrm{~h}$ entre l'état « naturel » et l'état actuel de prélèvement proche de $6 \mathrm{Mm}^{3}$. Pour ce qui concerne les crues de la saison été aucun impact n'a été identifié. Autrement dit, les éventuels pompages pendant la période d'irrigation n'influencent pas les crues de la saison été. Parallèlement à l'analyse des observations de débit et pour conforter l'impact anthropique observé sur les chroniques de crue, nous avons vérifié que l'aléa climatique, la pluie essentiellement, avait un comportement stationnaire ces trente dernières années.

L'absence d'impact sur les modules d'été $Q A \_E$ des sous-bassins de la Séoune et du Tescou est cohérente avec les résultats trouvés pour les crues d'été qui assurent pour une grande part la ressource des modules d'été. Pour ce qui concerne les modules d'hiver $Q A \_H$ des sous-bassins de la Séoune et du Tescou le coefficient d'écoulement moyen d'hiver diminue respectivement de $31 \%$ en moyenne et de $42 \%$ en moyenne entre l'état « naturel » et l'état anthropisé actuel. Cette diminution qui fait référence a une pluie annuelle moyenne d'hiver de $503 \mathrm{~mm}$ (1969-2000) pour le sous-bassin de la Séoune et de $465 \mathrm{~mm}$ (1975-2000) pour le sous-bassin du Tescou induit respectivement un déficit d'environ $30 \mathrm{Mm}^{3}$ et de $18,5 \mathrm{Mm}^{3}$ sans commune mesure avec les volumes théoriques stockés à partir des retenues collinaires. Autrement dit, les retenues collinaires ne peuvent expliquer à elles seules la forte diminution des coefficients d'écoulement observés et validée à partir des modélisations effectuées. Par ailleurs, la tendance observable sur les coefficients d'écoulement n'est pas le fait des pluies d'hiver correspondantes qui se sont révélées stationnaires sur la période d'observation des débits. Cela nous incite à retenir ces résultats pour des objectifs de gestion immédiats et ultérieurs de la ressource, si les conditions climatiques et d'occupation du sol n'évoluent guère.

II ne semble pas y avoir d'impact des éventuels pompages sur les débits d'étiage $\mathrm{VCN}_{30}$ "débit moyen minimum annuel de 30 jours consécutifs " en particulier pour la norme $V_{C N} N_{30}(5)$ des sous-bassins du Tescou et de la Séoune. De manière générale, la plus ou moins grande sévérité des étiages des deux sous-bassins dépend essentiellement de la variabilité climatique inter-saisonnière. Pour le sous-bassin du Tescou, le soutien d'étiage prévu de $2 \mathrm{Mm}^{3}$ est bénéfique puisqu'il permettrait un glissement en fréquence des quantiles secs vers les quantiles humides.

En définitive, seul le régime hydrologique d'hiver des sous-bassins se trouve affecté par l'usage de l'eau pour l'irrigation. La stationnarité des régimes hydrologiques d'été tendrait à montrer que l'incidence sur la ressource des éventuels pompages serait faible et par conséquent non détectable à partir des tests de ruptures de stationnarité appliqués aux chroniques hydrologiques et pour lesquels une réflexion théorique se poursuit (RENARD, 2004-2006). La satisfaction des besoins en eau pour l'irrigation dépendrait donc essentiellement du volume d'eau stocké par les retenues collinaires pendant la saison hiver.

Enfin, notre objectif à terme serait de prédire l'évolution du régime hydrologique naturel des bassins à aménager ou en cours d'aménagement, ce qui impliquerait à la fois une bonne connaissance régionale des régimes naturels et des régimes anthropisés. 


\section{REMERCIEMENTS}

Nous remercions toutes les personnes qui ont bien voulu contribuer à ce travail et tout particulièrement M. Michel BouzIGES de la DIREN Midi-Pyrénées, M. Bernard GAILLARD de la DIREN Aquitaine et M. Laurent BERTHELOT du Conseil Général du Tarn-et-Garonne.

\section{RÉFÉRENCES BIBLIOGRAPHIQUES}

BOIS P., 1986. Contrôle des séries chronologiques corrélées par étude du cumul des résidus. Deuxièmes journées hydrologiques de l'Orstom, p 89-100.

CACG, 2001. Comportement de la ressource en eau sur le bassin du Tescou : Rapport Final, 70 pages.

DELBREILH N., 1993. Approche de l'impact sur l'environnement d'un ensemble de retenues collinaires. Cas du bassin de la Séoune. Mémoire de DEA d'écologie des systèmes aquatiques continentaux de l'université $P$. Sabatier de Toulouse, 65 pages.

DIRECTIVE 2000/60/CE du Parlement Européen et du Conseil du 23 octobre 2000. Établit un cadre pour une politique communautaire dans le domaine de l'eau, Journal officiel des Communautés européennes, 22/12/2000, L 327, pages 172.

HOSKING J.R., WALLIS J.R., WOOD E.F., 1985. Estimation of the generalized extreme value distribution by the method of probability-weighted moment. Technometrics, 27(3) : 251-261.

GALÉA G. ET PRUDHOMME C., 1994. Modèles débit-durée-fréquence et conceptualisation d'un hydrogramme de crue synthétique: validation sur le BVRE de DRAIX - Hydrologie Continentale, 9 , $2: 139-151$.

JAVELLE P., GRÉSILLON JM. ET GALÉA G., 1999. Modélisation des courbes débitdurée-fréquence en crues et invariance d'échelle. Comptes-rendus de l'Académie des Sciences Paris, Sciences de la terre et des planètes, $329: 39-44$.
JAVELLE, P., GALÉA, G., GRÉSILLON, J.M., 2000. L'approche débit-durée-fréquence, historique et avancées, Revue des Sciences de l'Eau, 13(3) : 305-323.

JAVELLE P., 2001. Caractérisation du régime des crues: le modèle débit-durée-fréquence convergent, approche locale et régionale. Thèse de doctorat INPG (MMGE), UR Hydrologie-Hydraulique, Cemagref, groupement de Lyon, 268 pages.

LANG M., 1995. Les chroniques en hydrologie - modélisation comparée par un système de gestion de bases de données relationnel et orienté-objet. Traitements de base et intervalles de confiance des quantiles de crues. Techniques d'échantillonnage par la méthode du renouvellement. Thèse de doctorat, Université Joseph Fourier Grenoble, Cemagref, Groupement de Lyon, Division Hydrologie-Hydraulique, Ministère de l'Équipement, 296 pages.

LANG M., OUARDA T., BOBÉE B., 1999. Towards operational guidelines for overthreshold modelling. Journal of Hydro$\log y, 225: 103-117$.

LANG M., RENARD B., DINDAR L., LEMAITRE F., BOIS P., 2004. Use of a statistical test based on Poisson Process for the detection of changes in peakover-threshold series. Hydrology: Science and Practice for the 21 st. Century, Volume I, British Hydrological Society.

PERRIN, C., 2002. Vers une amélioration d'un modèle global pluie-débit au travers d'une approche comparative. La Houille Blanche, $n^{\circ}$ 6/7 : 84-91. 
RENARD B., 2004-2006 : Analyses statistiques pour la détection de tendances ou de ruptures dans le régime des crues et sécheresses en France. Thèse de doctorat en cours UR HydrologieHydraulique, Cemagref Groupement de Lyon.
VASQUEZ-PAULUS S., 2004. L'usage de l'eau en agriculture, ses conséquences sur le régime hydrologique des sousbassins du Tescou et de la Séoune (Bassin Adour-Garonne). Master-2 Recherche STUE (DEA), INP Grenoble, Université J. FOURIER, 74 pages. 